\title{
INVENTORY DECISION FOR PRODUCTS WITH DETERIORATION AND EXPIRATION DATES FOR POLLUTION-BASED SUPPLY CHAIN MODEL IN FUZZY ENVIRONMENTS
}

\author{
Mukunda Choudhury ${ }^{1}$, Sujit Kumar De² and Gour Chandra Mahata ${ }^{1, *}$
}

\begin{abstract}
The proposed study addresses a two-echelon sustainable supply chain (SC) model with a single-vendor and a single-buyer by considering the detrimental impacts of environmental pollution due to production. Moreover, an estimation function of pollution measure due to production is developed through a separate modelling. In the entire supply chain, we assume the deterioration rate increases with time and it also depends on the product's expiration date. On the other hand, the demand for deteriorating items at the buyer's end is assumed to be the dense fuzzy number because of learning effect. The model is developed by defining the exact profit functions for the vendor, the buyer and the entire supply chain and solved by classical method. These lead to the determination of individual optimal policies, as well as the optimal policy for the joint integrated supply chain. Fuzzifying the final objective function via dense fuzzy rule, we have employed extended ranking procedure for its defuzzification. A comparative study on numerical illustration of the proposed objective function under centralized and decentralized policies in both crisp and dense fuzzy environment has also been studied to validate the model. Finally graphical illustrations and sensitivity analysis have been made for its global justifications.
\end{abstract}

Mathematics Subject Classification. 90B05.

Received November 26, 2020. Accepted February 5, 2022.

\section{INTRODUCTION}

In present global production-supply scenario, green environment sustainability is the essential key factor for the existence of any kind of enterprise. Inventory management studies should consider the entire supply chain membership and establish an integrated inventory model, rather than adopting a retailer's or manufacturer's explicit model. The application of the production-inventory model to manufacturers and retailers in supply chain may be discussed in the following two ways.

Keywords. Supply chain, inventory, expiry date, dense fuzzy set, learning effect.

1 Department of Mathematics, Sidho-Kanho-Birsha University, Purulia 723104, West Bengal, India.

2 Department of Mathematics, Midnapore College (Autonomous), Paschim Medinipur 721101, West Bengal, India.

*Corresponding author: gourmahata@yahoo.co.in; gcmahata.skbu@gmail.com

(C) The authors. Published by EDP Sciences, ROADEF, SMAI 2022

This is an Open Access article distributed under the terms of the Creative Commons Attribution License (https://creativecommons.org/licenses/by/4.0), which permits unrestricted use, distribution, and reproduction in any medium, provided the original work is properly cited. 


\subsection{Early-stage inventories and supply chains}

Goyal [12] first developed an integrated inventory model based on the economic order quantity (EOQ) model to determine the optimal joint inventory policy for a single vendor and single buyer. Banerjee [2] developed a joint economic-lot-size model, in which a vendor produces to order for a buyer on a "lot-for-lot" basis. Goyal [13] relaxed the assumption of the "lot-for-lot" policy and got the better result from Banerjee [2]. Lu [28] subsequently extended Goyal's [13] model to consider a one-vendor and multiple buyers in an integrated inventory model and relaxed the assumption that shipments cannot be triggered until the entire production batch is completed. Goyal [14] further aimed Lu's [28] model to suggest that the shipment size to the buyer of production batch should be increased by a fixed factor. Hill [20] generalized the model of Goyal [14] by taking the geometric growth factor as a decision variable and demonstrated numerically on both equal shipment size policy and the policy adopted by Goyal [14]. Goyal and Nebebe [16] tried to exploit the benefits of equal-size and geometric policies and suggested a simple geometric-then-equal policy that produced positive results. Kelle et al. [25] first proposed production and shipping policy where the buyer's order is delivered in $n$ shipments of equal size and the vendor's production lot size can also be an integer $m$, multiple of the shipment size, where $m$ is different from $n$. Giri and Roy [11] developed production-inventory model in which the vendor offers a quantity discount to motivate the buyers to buy a larger quantity and delivered an unequal size per shipment. Thereafter, several researchers $([27,36,38,43]$ etc.) continued to propose additional ordering and shipping policies for integrated inventory models.

However, previous studies of production-inventory models only explored the production, transportation or ordering strategies, or all strategies together, but did not consider the effect of deterioration with expiration dates, environmental pollution due to production, uncertain demand for deteriorating items at the buyer's, and order sales which is necessary for a complete supply chain system. We know, the items, such as milk, fruit, blood, pharmaceutical product, vegetables etc. decay or deteriorate over time and similarly, COVID-19 materials can pollute more. Therefore, it is important to study the behavior of such decaying, deteriorating and pollution sensitive items, towards the formulation of appropriate inventory control policies that explicitly take such behavior into the account. Ghare and Schrader [10] were the first authors to consider the effect of decay on inventory items. They used the term "inventory decay" to describe this phenomenon, including direct spoilage, physical depletion and deterioration. They developed a general economic order quantity (EOQ) type model under constant demand with exponential decay, which could be solved iteratively, but not directly. Thereafter, a great deal of research efforts has been devoted to inventory models of deteriorating items, the details can be found in the review articles by Raafat [40] and Goyal and Giri [15]. Although numerous studies have been conducted on deterioration items, but few of them have been considered expiration (maximum lifetime) issue of deteriorating items. Mukherjee and Mahata [35] discussed about fixed lifetime of deteriorating items. Recently, Mahato and Mahata [32], Wang et al. [44], Wu et al. [46] discussed deteriorating items with maximum lifetime. Most existing inventory models for deteriorating items are EOQ models having different sub-systems in the supply chain independently. Although the notion of cooperation between suppliers and buyers has received more and more attention in the literature, but out of them few integrated inventory approaches for deteriorating items have been developed yet. Rau et al. [41] developed a multi-echelon inventory model for a deteriorating item and derive the optimal joint total cost from an integrated perspective, including the supplier, the producer and the buyer. In two-layer SC, pricing and quality discounts are also analyzed [21,37].

To consider the issues of pollution in inventory modelling very few research articles have been found in the literature. Most of them came from the policies of carbon footprint or carbon cap taxation also. The carbon constrained EOQ model was established by Hua et al. [23], He et al. [19] and Hovelaque and Bironneau [22]. However, Benjaafar et al. [4] argued that businesses can reduce carbon emissions considerably through operations management (such as inventory management) without substantially increasing their costs. He et al. [18] studied production lot-size model with carbon emissions under cap-and trade and carbon tax regulations. Mahato and Mahata [18] discussed all the potential cases, which may occur in the green inventory models with carbon emission cost under different allowable delay-in-payments. Recently, Shen et al. [42] explored the production 
and inventory problem in supply chain subject to a carbon tax policy. In all the above models, researchers have only considered all the parameters and variables as crisp values. Although crisp models offer an overview of the approach of inventory systems under various assumptions, they are not able to provide factual terms. As a result, exerting crisp models in general can lead to errors in decision-making. But, in crisp models, inventory managers must be flexible in determining the optimum economic lot size to cause non-random uncertainty-based cost reduction in practice.

\subsection{Modern supply chains and various approaches}

Zadeh [49] developed the concepts of fuzzy sets to capture the uncertainties in decision making. Since then, Bellman and Zadeh [3] applied it in decision making for industrial management problems. Mahata and Goswami [30] proposed an EOQ model for deteriorating items considering the fuzzy cost parameters. Mahata and Mahata [31] studied a fuzzy EOQ inventory model with two phase trade credits for deteriorating items in the fuzzy sense. In another study, Mahata [29] discussed an imperfect production model with partial backlogging of shortage quantity in fuzzy random environments. De and Sana [8] studied a hill type fuzzy stochastic model and got solutions via Bonferroni mean operator over score function of the fuzzy numbers.Type-2 fuzzy set has been utilized for solving four-layer green supply chain model [39]. Also, to solve a backorder model nonlinear heptagonal dense fuzzy set is employed [34].

However, in the literature several ranking rules have been adopted by the eminent researchers like Yager [47], Allahviranloo and Saneifard [1], Ezzati et al. [9], etc. The concepts of deviation degree in fuzzy numbers were hosted by Kumar et al. [26], Hajjari and Abbasbandy [17], Yu et al. [48], etc. in developing the several inventory models. The main key factor of an inventory under smooth running is the supply of demand to the customers as quick as possible. But in reality, we see due to lack of information, the decision makers used go for wrong decision in management system. So, information gathering is one of the most essential part and parcel of any SC process. In the literature, attempts have been taken by Kazemi et al. [24] to gain information through learning and for getting process in fuzzy parameters for the backorder EOQ model with imperfect quality items. They applied Wright's [45] learning curve to gain knowledge in which the numbers of shipments are the vital factor. In another study, De and Mahata [7] discussed the learning effect on demand parameter in a backorder EOQ model through the variation of cycle time. Their basic notion is that, longer cycle time of an inventory could motivate customers in favor of that inventory spontaneously. They used the cloudy fuzzy approach on demand and compared the results with that of the general fuzzy model to justify their new approach. But in our study, we have shown that customers/public interactions with the decision makers (DM) can change the motivation so that a catchment area over demand reaches very soon. In this study we have shown that adequate interaction could perform better goal in favor of inventory management system. However, a reverse logic, more and more interactions of the DM to their learned customers can make a harmful situation (non-favorable to DM) on the process itself. Also, such adequacy on interactions/negotiations/bargaining may vary from commodity to commodity, situations to situations even customer to customer or customer to DM implicitly. Thus, we take the customer's demand as dense fuzzy number to estimate the actual learning outcomes in the inventory process itself. We have utilized De and Beg's [6] ranking index rule to defuzzify the fuzzy objective function.

Thus, it is seen that, none of the authors has presented an optimal production, shipping, ordering, and environmental pollution due to production, uncertain demand for deteriorating items at the buyer's site for an integrated supply chain system with respect to deteriorating raw materials and finished products with expiration date. No study has yet been considered over a two-echelon supply chain considering the detrimental impacts of deterioration using environmental pollution due to production also. The deterioration rate increases with time and it also depends on the product's expiration date. Further, the demand for deteriorating items at the buyer's end is assumed to be the dense fuzzy number because of learning effect. In terms of model formulation, the total profit functions of the retailer and manufacturer are established and then integrated to obtain the joint total profit function per unit time. Moreover, we split the model into two different cases namely crisp and dense fuzzy environments. Applying ranking index rule the new expressions for decision variables are developed. Numerical 
examples are also studied extensively. A sensitivity analysis and graphical illustrations are done to justify the new approach. At the end, a conclusion is made followed the scope of future work.

Now, we may put the basic research questions over here:

(a) Is it possible to estimate the environmental pollution due to production in two-layer supply chain?

(b) What will be the optimal order quantity of the buyer, the optimum cycle time for vendor and buyer and their corresponding profit values?

(c) What is the learning frequency value whenever the demand rate assumes dense fuzzy number under nonrandom uncertain system?

(d) Whether the centralized policy could be able to create more profit of the SC or not.

\section{Preliminary}

In this section we shall discuss some useful concepts and definitions which are frequently used in developing this model.

\subsection{Triangular dense fuzzy set (TDFS) [6]}

The fuzzy set of the form

$$
\tilde{A}=\left\langle a_{2}\left(1-\frac{\rho}{1+k}\right), a_{2}, a_{2}\left(1+\frac{\sigma}{1+k}\right)\right\rangle, \quad \text { for } 0<\rho, \sigma<1
$$

is called TDFS where $k$ represents the learning frequency parameter, $\rho, \sigma$ are the fuzzy left and right deviation parameters and $a_{2}$ is the crisp/classical value. The memberships function for $0 \leq k$ is defined as follows:

$$
\mu(x, k)= \begin{cases}0, & \text { if } x<a_{2}\left(1-\frac{\rho}{1+k}\right) \text { and } x>a_{2}\left(1+\frac{\sigma}{1+k}\right) \\ \frac{x-a_{2}\left(1-\frac{\rho}{1+k}\right)}{\frac{\rho a_{2}}{1+k}}, & \text { if } a_{2}\left(1-\frac{\rho}{1+k}\right) \leq x \leq a_{2} \\ \frac{a_{2}\left(1+\frac{\sigma}{1+k}\right)-x}{\frac{\sigma a_{2}}{1+k}}, & \text { if } a_{2} \leq x \leq a_{2}\left(1+\frac{\sigma}{1+k}\right) .\end{cases}
$$

\subsection{Defuzzification method based on $\alpha$-cuts}

The left and the right $\alpha$-cuts of a triangular dense fuzzy number $\tilde{A}=\mu(x, k)$ are, $L^{-1}(\alpha, k)=$ $a_{2}\left(1-\frac{\rho}{1+k}+\frac{\rho \alpha}{1+k}\right)$ and $R^{-1}(\alpha, n)=a_{2}\left(1+\frac{\sigma}{1+k}-\frac{\sigma \alpha}{1+k}\right)$. So,

$$
\begin{aligned}
L^{-1}(\alpha, k)+R^{-1}(\alpha, k) & =a_{2}\left(2-\frac{\rho}{1+k}+\frac{\rho \alpha}{1+k}+\frac{\sigma}{1+k}-\frac{\sigma \alpha}{1+k}\right) \\
& =a_{2}\left\{2+\frac{(\rho-\sigma) \alpha}{1+k}+\frac{\sigma-\rho}{1+k}\right\} .
\end{aligned}
$$

Now, we use the Yager's [47] method of ranking index as an extension of the new defuzzification method for the TDFS $\tilde{A}$ and it is stated as follows:

$$
I(\tilde{A})=\frac{1}{2 N} \sum_{k=0}^{N} \int_{0}^{1}\left\{L^{-1}(\alpha, k)+R^{-1}(\alpha, k)\right\} \mathrm{d} \alpha .
$$

Thus,

Hence

$$
\int_{0}^{1}\left\{L^{-1}(\alpha, k)+R^{-1}(\alpha, k)\right\} \mathrm{d} \alpha=a_{2}\left\{2+\frac{\sigma-\rho}{2(1+k)}\right\}
$$

$$
I(\tilde{A})=\frac{1}{2 N} \sum_{k=0}^{N} a_{2}\left\{2+\frac{\sigma-\rho}{2(1+k)}\right\}=\frac{a_{2}}{2 N}\left[2 N+\frac{\sigma-\rho}{2}\left\{\frac{1}{1+0}+\frac{1}{1+1}+\frac{1}{1+2}+\ldots+\frac{1}{1+N}\right\}\right] .
$$




\section{Notation AND ASSUMPtions}

\subsection{Notations}

The following notation is used throughout this paper for the development of the production-inventory model:

$A_{b} \quad$ Buyer's ordering cost per replenishment cycle $(\$)$

$A_{v} \quad$ Vendor's setup cost per production cycle $(\$)$

$c \quad$ Vendor's product cost per unit $(\$)$

$v \quad$ Vendor's supply price per unit $(\$)$

$s \quad$ Buyer's selling price per unit $(\$)$

$h_{b} \quad$ Buyer's holding cost per unit per unit time $(\$)$

$h_{v} \quad$ Vendor's holding cost per unit per unit time $(\$)$

$c_{T} \quad$ Buyer's fixed shipping cost per shipment (\$)

$c_{t} \quad$ Buyer's variable shipping cost per unit $(\$)$

$p_{c} \quad$ Pollution cost per unit pollution index $(\$)$

$\theta \quad$ Deterioration rate of the item per unit quantity

$L \quad$ Expiration date of the item (year)

$P \quad$ Vendor's production rate (unit/year)

$D \quad$ Buyer's demand rate (unit/year)

$F(t) \quad$ Freshness index of the item in time $t$ (a function of the expiration date)

$T_{p} \quad$ Time length between the first production and shipping items from the vendor to the buyer

$T_{b} \quad$ Length of the buyer's replenishment cycle time, a decision variable

$T_{v} \quad$ Length of the vendor's production time, a decision variable

$m \quad$ Number of shipments from the vendor to the buyer, a decision variable

$Q \quad$ Buyer's order quantity, a decision variable

$q \quad$ Shipped quantity from the vendor to the buyer on each occasion, $q=\frac{Q}{m}$

\subsection{Assumptions}

(i) The production-inventory system involves a single vendor, single buyer, and single commodity.

(ii) The vendor's production rate is finite. During production period $P(1-\theta)>D$ is satisfied.

(iii) The inventory system involves one type of deteriorating item with expiration date $L$, and both quantity and quality losses are involved.

(iv) Deterioration occurs in vendor's and buyer's inventory. By referring to Mahato and Mahata [32], Wu et al. [46] and Mukherjee and Mahata [35], during the expiration date $L$, the quantity loss rate of the items can be defined as follows:

$$
\theta(t)=\frac{1}{1+L-t}, \quad 0 \leq t \leq T_{b} \leq L
$$

(v) The buyer orders a lot of size $Q$ units and requires the vendor to divide $n$ consignments and to deliver $q$ units in each shipment. All shipping costs are borne by the buyer.

(vi) Production rate and demand rate are independent of the production lot size and are constant.

(vii) Shortages are not permitted for the vendor or buyer.

\section{Model FORMUlation}

In this paper, we investigate an integrated inventory model for deteriorating items with expiration date considering environmental pollution. First, we formulated the following simple description of the productioninventory system: during an entire production cycle (the length of the period is $T_{v}$ ) in the supply chain system, the buyer orders $Q$ units, which the vendor is then required to divide into $n$ consignments. The number of deliveries is $q=\frac{Q}{m}$ units per shipment. In compliance with the JIT system, the vendor begins shipment during the production period and ships to the buyer when the production quantity first reaches $q$ units (the length of the period is $T_{p}$ ). The vendor then ships $q$ units at the expected time (the length of the period is $T_{b}$ ). Because 
the production rate is greater than the demand rate, the vendor will cease production and continue to make regular shipments until the total quantity shipped reaches $I_{\max }$ (the length of the period is $T_{s}$ ). Based on the aforementioned notation and assumptions, we first establish the total profit function per unit of time for the buyer and vendor as follows.

\subsection{Estimation of pollution level due to production}

Here we shall discuss the story behind environmental pollution and its assessment. Traditionally, researchers are engaged in developing various models of producing commodities at different industrial sectors; its transportation and supply chains. We know, for any kind of industrial production there corresponds a considerable amount of pollution which are adding to the environment day by day. Also, human intervention upon environment is creating environmental hazards and pollution accumulation (the collective effect of air, soil and water pollution). Such kind of "pollution-production" has no outlet to discharge/transfer or in other words this produced item has no demand. However, in practical situation we found a little bit of its reduction by means of absorption by nature (environment). For example, small particulate matters of air are getting washed out during rain fall, the green vegetation absorbs $\mathrm{CO}_{2}$ during photosynthesis, the harmful pollutants like $\mathrm{SO}_{2}, \mathrm{CO}, \mathrm{SO}_{3}$ etc. are converted to some other substances with the use of technological advancement associated to production industries itself.

Therefore, we may design a pollution production model under some assumptions and notations exclusively.

\subsubsection{Assumptions and notations}

\section{Assumptions}

(i) The environment itself has a minimum level of pollution whether human intervention upon environment takes place or not.

(ii) $100 \%$ pollution reduction is not possible; rather a little part of total pollution can be reducible with the help of advanced technology and by the vegetation of environment and hence large amount of pollution is getting pile up over time.

(iii) Amount of pollution adding to the environment follows geometric progression.

(iv) At the time of infinity, the pollution level reaches to a maximum level, called life limit.

(v) All kinds of pollutions are harm to animal and plant kingdom.

(vi) Amount of pollution means the average of $m$ successive amount of pollution came from $n$ production cycles of an industry.

(vii) The term "life limit" means a certain amount of environmental pollution for which about $80 \%$ each of the animal and plant species might extinct of.

\section{Notations \\ $J_{i} \quad$ Pollution level at any time $t$ of the $i$-th production cycle. \\ $p \quad$ Rate of pollution absorption by the nature. \\ $p_{0}^{i-1} \quad$ The threshold amount of pollution at the $i$-th production cycle. \\ $w_{m} \quad$ Total amount of pollution after $n$ production cycle. \\ $T$ The industrial production cycle time (months) \\ $\lambda^{\prime}, \lambda$ Scale parameters of pollution \\ $p^{i} \quad$ Pollution rate at the $i$-th production cycle}

\subsubsection{Mathematical foundation of Pollution Production (PP) model}

Let the pollution rate $\lambda p^{i}$ is adding to the environment during the $i$-th industrial production cycle of a single industrial set up (Fig. 1). The pollution is reduced due to environmental absorption only and the major parts are adding to the environment over time. Then considering the above assumptions and for $n$ cycles of production 


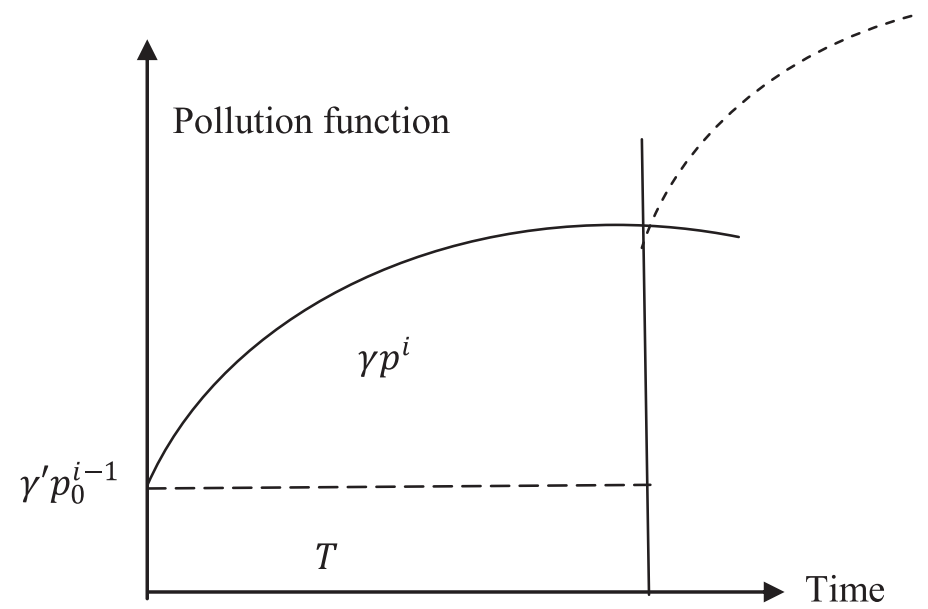

Figure 1. Pollution generating model at $i$ th production process.

the governing differential equation of the pollution production model is given by

$$
\begin{cases}\frac{\mathrm{d} J_{i}(t)}{\mathrm{d} t}+\varphi J_{i}(t)=\gamma p^{i}, & 0 \leq t<\infty \\ \text { Subject to } J_{i}(0)=\gamma^{\prime} p_{0}^{i-1}, & i=1,2,3, \ldots, m\end{cases}
$$

Solving (4.1) we get

$$
J_{i}(t)=\gamma^{\prime} p_{0}^{i-1} e^{-\varphi t}+\gamma p^{i}\left(1-e^{-\varphi t}\right) .
$$

Now utilizing (4.2) the total amount of pollution after $m$ cycles is given by

$$
w_{m}=\sum_{i=1}^{m} \int_{0}^{m T} J_{i}(t) \mathrm{d} t=\int_{0}^{m T}\left\{\gamma^{\prime} e^{-\varphi t} \sum_{i=1}^{m} p_{0}^{i-1}+\gamma\left(1-e^{-\varphi t}\right) \sum_{i=1}^{m} p^{i}\right\} \mathrm{d} t .
$$

So average amount of cumulative pollution per cycle over $m$ cycles is

$$
\bar{w}=\frac{w_{m}}{m}=\left[\left\{T-\frac{\left(1-e^{-\varphi n T}\right)}{m \varphi}\right\} \frac{\gamma p\left(p^{m}-1\right)}{p-1}+\frac{\left(1-e^{-\varphi m T}\right)}{m \varphi} \frac{\gamma^{\prime}\left(p_{0}^{m}-1\right)}{p_{0}-1}\right] .
$$

So, the total pollution cost is given by

$$
\mathrm{PLC}=p_{c}\left[\left(m T_{v}-\frac{\left(1-e^{-\varphi m T_{v}}\right)}{\varphi}\right) \frac{\gamma p\left(p^{m}-1\right)}{p-1}+\frac{\left(1-e^{-\varphi m T_{v}}\right)}{\varphi} \frac{\gamma^{\prime}\left(p_{0}^{m}-1\right)}{p_{0}-1}\right] .
$$

\section{Special cases}

(i) When the natural absorption rate $\varphi \rightarrow 0$ then $\bar{w} \rightarrow T \frac{\gamma^{\prime}\left(p_{0}^{m}-1\right)}{p_{0}-1}$.

[Since,

$$
\begin{aligned}
\lim _{\varphi \rightarrow 0} \bar{w} & =\lim _{\varphi \rightarrow 0} \frac{1}{m}\left[\left\{m T-\frac{\left(1-e^{-\varphi m T}\right)}{\varphi}\right\} \frac{\gamma p\left(p^{m}-1\right)}{p-1}+\frac{\left(1-e^{-\varphi m T}\right)}{\varphi} \frac{\gamma^{\prime}\left(p_{0}^{m}-1\right)}{p_{0}-1}\right] \\
& =\lim _{\varphi \rightarrow 0} \frac{1}{m}\left[\left\{m T-\frac{m T\left(e^{-\varphi m T}-1\right)}{-m \varphi T}\right\} \frac{\gamma p\left(p^{m}-1\right)}{p-1}+\frac{\left(e^{-\varphi m T}-1\right) m T}{-m \varphi T} \frac{\gamma^{\prime}\left(p_{0}^{m}-1\right)}{p_{0}-1}\right]
\end{aligned}
$$




$$
=\frac{T \gamma^{\prime}\left(p_{0}^{m}-1\right)}{p_{0}-1}
$$

So, $\bar{w} \rightarrow T \frac{\gamma^{\prime}\left(p_{0}^{m}-1\right)}{p_{0}-1}$. ]

(ii) When the natural absorption rate $\varphi \rightarrow \infty$ then $\bar{w} \rightarrow T \frac{\gamma p\left(p^{m}-1\right)}{p-1}$. [Since, $\lim _{\varphi \rightarrow \infty} \bar{w}=$ $\lim _{\varphi \rightarrow \infty} \frac{1}{n}\left[\left\{m T-\frac{\left(1-e^{-\varphi m T}\right)}{\varphi}\right\} \frac{\gamma p\left(p^{m}-1\right)}{p-1}+\frac{\left(1-e^{-\varphi m T}\right)}{\varphi} \frac{T \gamma^{\prime}\left(p_{0}^{m}-1\right)}{p_{0}-1}\right]$.

Now, $\lim _{\varphi \rightarrow \infty} \frac{\left(1-e^{-\varphi m T}\right)}{\varphi}=\lim _{\varphi \rightarrow \infty} \frac{\left(e^{\varphi m T}-1\right)}{\varphi e^{\varphi m T}}=\left(\frac{\infty}{\infty}\right)$ form. So, applying L' Hospital's rule we get

$$
\lim _{\varphi \rightarrow \infty} \frac{\left(1-e^{-\varphi m T}\right)}{\varphi}=\lim _{\varphi \rightarrow \infty}\left[\frac{m T e^{\varphi m T}}{e^{\varphi m T}+\varphi m T e^{\varphi m T}}\right]=\lim _{\varphi \rightarrow \infty}\left[\frac{m T}{1+\varphi m T}\right]=0 .
$$

\subsection{The Buyer's annual profit function}

The buyer's inventory model can be depicted as in Figure 2. Replenishment cycle is marked by the receipt of an order quantity $q$ from the vendor. This inventory is displayed on shelves at the retail outlet and it can only be kept for a specified amount of time, known as the expiration date. Once this date has elapsed, the inventory can no longer be used to meet consumers' demand. Over the replenishment cycle, the buyer's inventory level at time $t$ decreases due to the effect of both market demand and deterioration of the item and is governed by the following differential equation:

$$
\frac{\mathrm{d} I_{b}(t)}{\mathrm{d} t}+\frac{1}{1+L-t} I_{b}(t)=-D, \quad 0 \leq t \leq T_{b}
$$

Solving (4.4) with the boundary condition $I_{b}\left(T_{b}\right)=0$, we obtain the buyer's inventory level as

$$
I_{b}(t)=D(1+L-t) \log \left[\frac{1+L-t}{1+L-T_{b}}\right], \quad 0 \leq t \leq T_{b} .
$$

Given that the buyer receives an order quantity $q$ at the start of eachcycle (i.e., $t=0)$, the boundary condition $I_{b}(0)=q$ is binding. Through substitution in (4.5), it follows that

$$
q=I_{b}(0)=D(1+L) \log \left[\frac{1+L}{1+L-T_{b}}\right] .
$$

The buyer's total profit per replenishment cycle includes sales revenue (SR), ordering cost (OC), transportation cost (TPC), purchase cost (PC), holding cost (HC).

These components are evaluated as follows:

(i) Sales revenue (SR): during the replenishment cycle, the buyer's total sales volume per replenishment cycle is, $Q_{d}=\mathrm{DT}_{b}$. Thus, the buyer's sales revenue per replenishment cycle is, $\mathrm{SR}=s \mathrm{DT}_{b}$.

(ii) Ordering cost (OC): the buyer's ordering cost per replenishment cycle is, $\mathrm{OC}=A_{b}$

(iii) Purchase cost (PC): the buyer's purchase cost is, $\mathrm{PC}=v q=v D(1+L) \log \left[\frac{1+L}{1+L-T_{b}}\right]$.

(iv) Inventory holding cost (HC): the buyer's holding cost per replenishment cycle is,

$$
\mathrm{HC}=h_{b} \int_{0}^{T_{b}} I_{b}(t) \mathrm{d} t=h_{b} D\left[\frac{1}{2}\left(1+L^{2}\right) \log \left(\frac{1+L}{1+L-T_{b}}\right)+L \log \left(\frac{1+L}{1+L-T_{b}}\right)-\frac{1}{2} T_{b}-\frac{1}{2} L T_{b}+\frac{1}{4} T_{b}^{2}\right] .
$$

(v) Transportation cost (TC): the buyer's transportation cost including fixed and variable costs per replenishment cycle is,

$$
\mathrm{TPC}=c_{T}+c_{t} q=c_{T}+c_{t} D(1+L) \log \left[\frac{1+L}{1+L-T_{b}}\right] .
$$




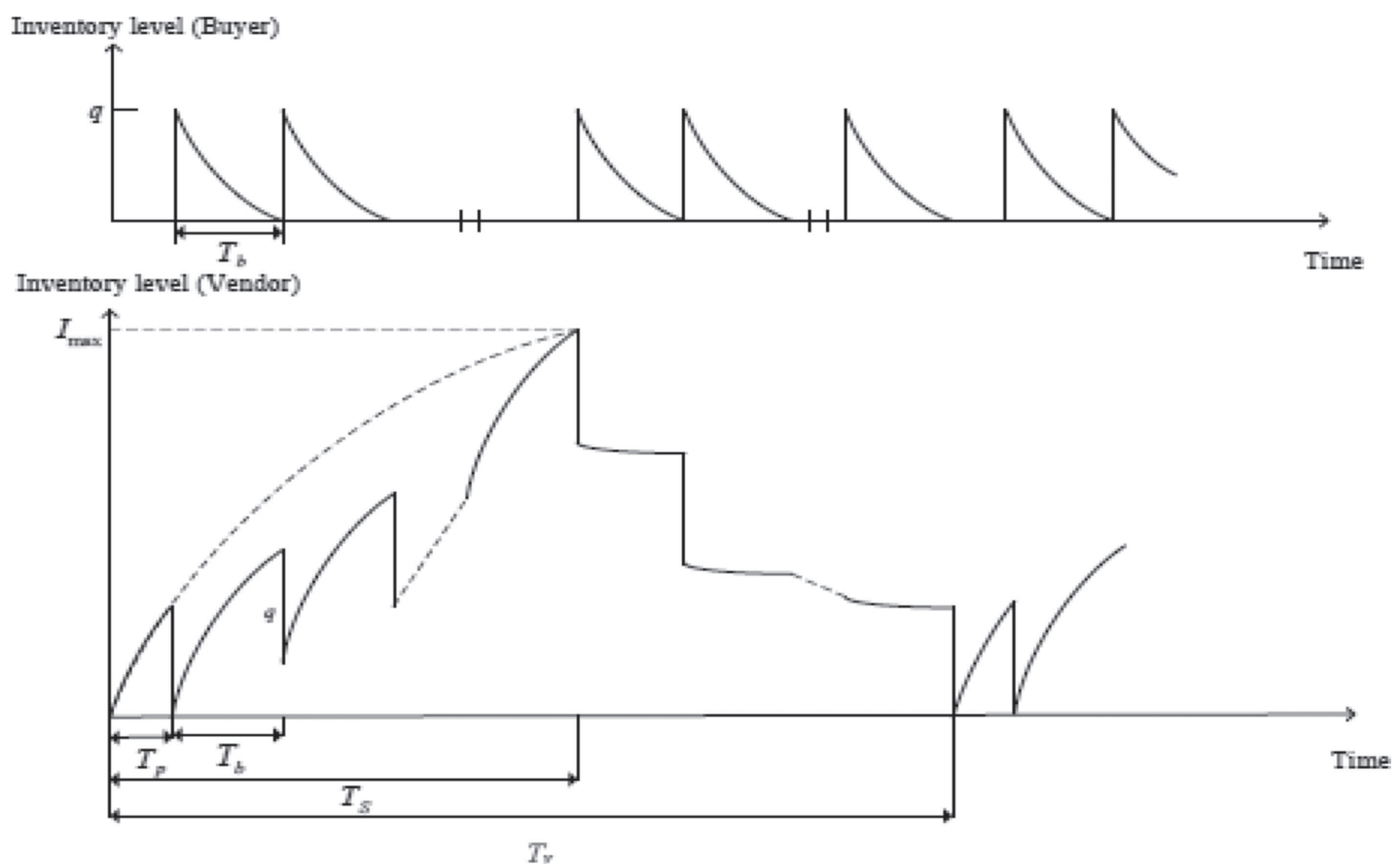

FiguRE 2. The vendor's and buyer's inventory systems in an entire production cycle.

Combining the above calculated cost components; we obtain the buyer's total profit per unit time as,

$$
\begin{aligned}
\operatorname{TP}_{b}\left(T_{b}\right)= & s D-\frac{1}{T_{b}}\left[A_{b}+c_{T}+\left(v+c_{t}\right) D(1+L) \log \left(\frac{1+L}{1+L-T_{b}}\right)\right] \\
& -\frac{h_{b}}{T_{b}} D\left[\frac{1}{2}\left(1+L^{2}\right) \log \left(\frac{1+L}{1+L-T_{b}}\right)+L \log \left(\frac{1+L}{1+L-T_{b}}\right)-\frac{1}{2} T_{b}-\frac{1}{2} L T_{b}+\frac{1}{4} T_{b}^{2}\right] .
\end{aligned}
$$

Proposition 4.1. Buyer's total profit per unit time $\mathrm{TP}_{b}\left(T_{b}\right)$ is a strictly pseudo-concave function in $T_{b}$, and hence exists a unique maximum solution $T_{b}=T_{b}^{*}$, where $T_{b}^{*} \in(0, \infty)$ satisfying $\frac{\mathrm{dTP}_{b}\left(T_{b}\right)}{\mathrm{d} T_{b}}=0$.

Proof. See Appendix A.

To find $T_{b}^{*}$, taking the first-order derivative of $\mathrm{TP}_{b}\left(T_{b}\right)$ with respect to $T_{b}$, setting the result to zero, we get

$$
\begin{aligned}
\frac{\mathrm{dTP}_{b}\left(T_{b}\right)}{\mathrm{d} T_{b}}= & \frac{1}{T_{b}^{2}}\left[A_{b}+c_{T}+\left(v+c_{t}\right) D(1+L) \log \left(\frac{1+L}{1+L-T_{b}}\right)\right]-\frac{\left(v+c_{T}\right) D(1+L)}{T_{b}\left(1+L-T_{b}\right)} \\
& +h_{b} D \frac{1}{T_{b}^{2}}\left[\frac{1}{2}\left(1+L^{2}\right) \log \left(\frac{1+L}{1+L-T_{b}}\right)+L \log \left(\frac{1+L}{1+L-T_{b}}\right)-\frac{1}{2} T_{b}-\frac{1}{2} L T_{b}+\frac{1}{4} T_{b}^{2}\right] \\
& -\frac{h_{b} D}{T_{b}}\left[\frac{(1+L)^{2}}{2\left(1+L-T_{b}\right)}+\frac{L}{\left(1+L-T_{b}\right)}-\frac{1}{2}-\frac{L}{2}+\frac{T_{b}}{2}\right]=0 .
\end{aligned}
$$

The expressions for necessary and sufficient conditions are complicated to obtain an analytical closed-form solution for the decision variable $T_{b}$ from equation (4.8). 


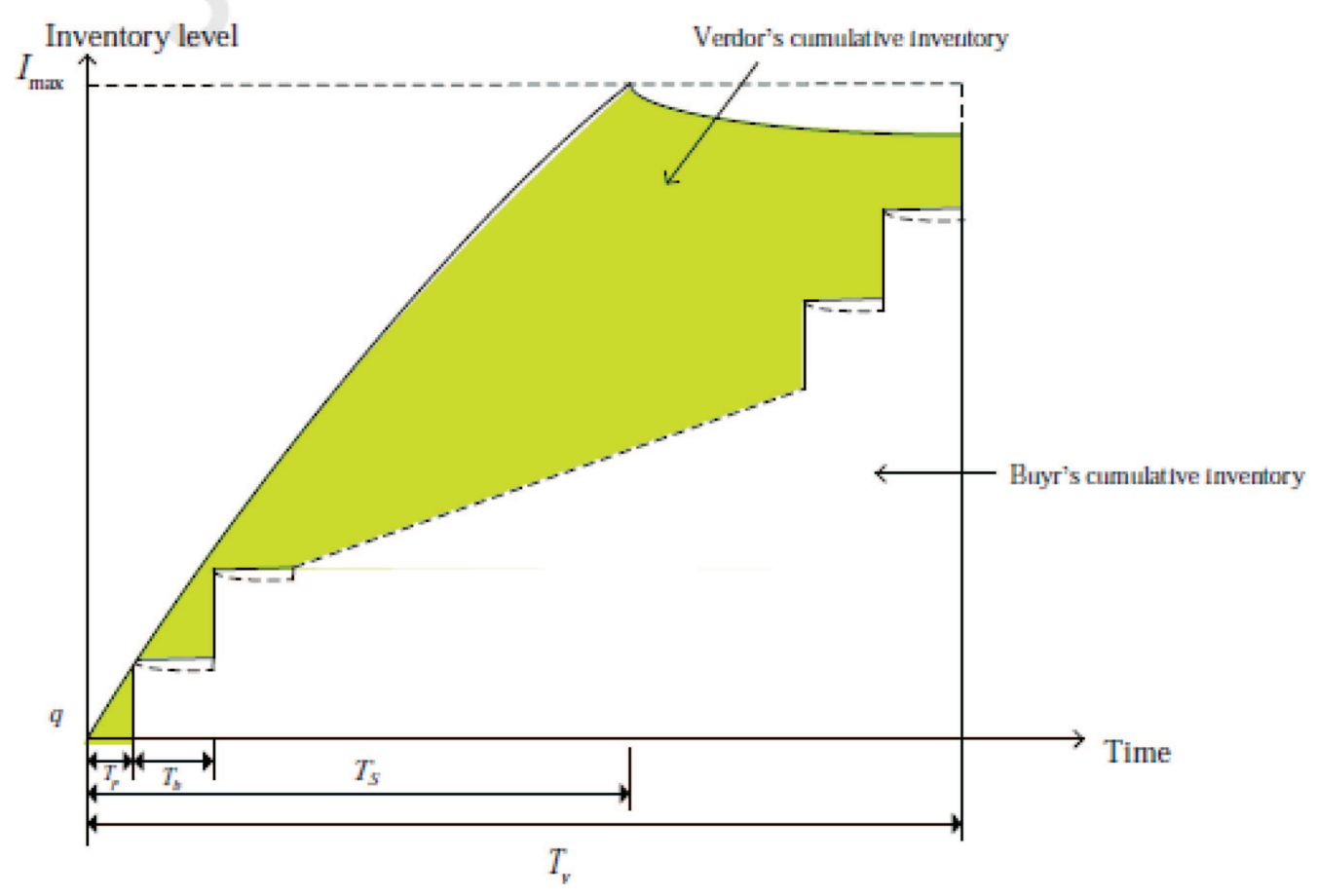

FiguRE 3. The vendor's and buyer's cumulative inventory.

\subsection{The vendor's annual profit function}

The vendor and buyer's cumulative inventory can be shown in Figure 3.

According to the vendor, during the time interval $\left[0, T_{s}\right]$, the inventory level of the vendor changes by the combined effect of production and deterioration of the item. Hence, the vendor's inventory level at time $t$ during the time interval $\left[0, T_{s}\right]$ is governed by the following differential equation:

$$
\frac{\mathrm{d} I_{p}(t)}{\mathrm{d} t}+\frac{1}{1+L-t} I_{p}(t)=P, \quad 0 \leq T \leq T_{s}
$$

with boundary condition $I_{p}(0)=0$. Solving (4.9), we obtain

$$
I_{p}(t)=P(1+L-t) \log \left(\frac{1+L}{1+L-t}\right), \quad 0 \leq T \leq T_{s} .
$$

From Figure $3, I_{p}\left(T_{p}\right)=q$ implies

$$
T_{p}=(1+L)\left[1-e^{-\frac{q}{p\left(1+L-T_{p}\right)}}\right]
$$

During the time interval $\left[T_{s}, T_{v}\right]$, the vendor's inventory level decreases because of item deterioration, and its inventory level at time $t$ during the time interval $\left[T_{s}, T_{v}\right]$ is governed by the following differential equation:

$$
\frac{\mathrm{d} I_{d}(t)}{\mathrm{d} t}+\frac{1}{1+L-t} I_{d}(t)=0, \quad T_{s} \leq T \leq T_{v}
$$


Similarly, the vendor's inventory level during the time interval $\left[T_{s}, T_{v}\right]$ with the boundary condition $I_{d}\left(T_{v}\right)=$ $m q=m D(1+L) \log \left[\frac{1+L}{1+L-T_{b}}\right]$ can be obtained by solving (4.12), which yields

$$
I_{d}(t)=m D(1+L) \log \left[\frac{1+L}{1+L-T_{b}}\right]\left(\frac{1+L-t}{1+L-T_{v}}\right), \quad T_{s} \leq T \leq T_{v} .
$$

From (4.10), (4.13), and the fact $I_{p}\left(T_{s}\right)=I_{d}\left(T_{s}\right)$, the following equation can be produced:

$$
T_{s}=(1+L)\left[1-e^{\frac{-m q}{P\left(1+L-T_{v}\right)}}\right] .
$$

Similarly, the vendor's total profit per production cycle includes sale revenue, setup cost, production cost, holding cost, and pollution cost. These components are evaluated as follows:

(i) The vendor's sales revenue per production cycle is,

$$
\mathrm{SR}_{v}=v Q=v m q=v m D(1+L) \log \left[\frac{1+L}{1+L-T_{b}}\right]
$$

(ii) The vendor's setup cost per production cycle is $A_{v}$

(iii) The vendor's production cost per production cycle is

$$
c P T_{s}=c P(1+L)\left[1-e^{\frac{-m q}{P\left(1+L-T_{v}\right)}}\right] .
$$

(iv) Holding cost which is as follows. The vendor's total inventory per production cycle is equal to the vendor's cumulative inventory minus the buyer's cumulative inventory, and is given by

$$
\int_{0}^{T_{s}} I_{p}(t) \mathrm{d} t+\int_{T_{s}}^{T_{v}} I_{d}(t) \mathrm{d} t-\left[q T_{b}(1+2+\ldots+(m-1))\right] .
$$

Therefore, the vendor's total holding cost per production cycle is

$$
\begin{aligned}
h_{v}[ & \left.\int_{0}^{T_{s}} I_{p}(t) \mathrm{d} t+\int_{T_{s}}^{T_{v}} I_{d}(t) \mathrm{d} t-\left[q T_{b}(1+2+\ldots+(m-1))\right]\right] \\
= & h_{v} P\left[\frac{\log (1+L)}{2}\left\{(1+L)^{2}-\left(1+L-T_{s}\right)^{2}\right\}\right] \\
& -h_{v} P\left[\left\{\frac{(1+L)^{2}}{2} \log (1+L)-\frac{(1+L)^{2}}{4}\right\}-\left\{\frac{\left(1+L-T_{s}\right)^{2}}{2} \log \left(1+L-T_{s}\right)-\frac{\left(1+L-T_{s}\right)^{2}}{4}\right\}\right] \\
& +h_{v} \frac{m q}{\left(1+L-T_{v}\right)}\left[\left\{(1+L) T_{v}-\frac{T_{v}^{2}}{2}\right\}-\left\{(1+L) T_{s}-\frac{T_{s}^{2}}{2}\right\}\right] \\
& -h_{v} T_{b} \frac{m(m-1)}{2} D(1+L) \ln \left[\frac{1+L}{1+L-T_{b}}\right] .
\end{aligned}
$$

(v) Pollution cost per production cycle is given by

$$
p_{c}\left[\left(m T_{v}-\frac{\left(1-e^{-\varphi m T_{v}}\right)}{\varphi}\right) \frac{\gamma p\left(p^{m}-1\right)}{p-1}+\frac{\left(1-e^{-\varphi m T_{v}}\right)}{\varphi} \frac{\gamma^{\prime}\left(p_{0}^{m}-1\right)}{p_{0}-1}\right] .
$$


Consequently, the vendor's total profit per unit of time (denoted by $\operatorname{TP}_{v}\left(m, T_{v}\right)$ is

$$
\begin{aligned}
\operatorname{TP}_{v}\left(m, T_{v}\right)= & \frac{1}{T_{v}+T_{b}}\left\{v m D(1+L) \log \left[\frac{1+L}{1+L-T_{b}}\right]-A_{v}-c P(1+L)\left[1-e^{\frac{-m q}{P\left(1+L-T_{v}\right)}}\right] .\right. \\
& -h_{v} P\left[\frac{\log (1+L)}{2}\left\{(1+L)^{2}-\left(1+L-T_{s}\right)^{2}\right\}\right] \\
& +h_{v} P\left[\frac{(1+L)^{2}}{2}\left\{\log (1+L)-\frac{1}{2}\right\}-\frac{1}{2}\left(1+L-T_{s}\right)^{2}\left\{\log \left(1+L-T_{s}\right)-\frac{1}{2}\right\}\right] \\
& -h_{v} \frac{m q}{\left(1+L-T_{v}\right)}\left[\left\{(1+L) T_{v}-\frac{T_{v}^{2}}{2}\right\}-\left\{(1+L) T_{s}-\frac{T_{s}^{2}}{2}\right\}\right] \\
& +h_{v} T_{b} \frac{m(m-1)}{2} D(1+L) \log \left[\frac{1+L}{1+L-T_{b}}\right] \\
& \left.-p_{c}\left[\left(m T_{v}-\frac{\left(1-e^{-\varphi m T_{v}}\right)}{\varphi}\right) \frac{\gamma p\left(p^{m}-1\right)}{p-1}+\frac{\left(1-e^{-\varphi m T_{v}}\right)}{\varphi} \frac{\gamma^{\prime}\left(p_{0}^{m}-1\right)}{p_{0}-1}\right]\right\} .
\end{aligned}
$$

Due to the fact that $T_{v}=T_{p}+(m-1) T_{b}$ from Figure $3, \operatorname{TP}_{v}\left(m, T_{v}\right)$ can be reduced to $\operatorname{TP}_{v}(m)$.

Proposition 4.2. Vendor's total profit per unit time $\mathrm{TP}_{v}(m)$ is strictly pseudo-concave in $m$, and hence exists a unique maximum solution at the point $m=m^{*}$, where $m^{*} \in(0, \infty)$.

Proof. See Appendix B.

Taking the first-order derivative of $\operatorname{TP}_{v}(m)$ in (4.15) with respect to $m$, setting the result to zero, we get

$$
\frac{\mathrm{dTP}_{v}(m)}{\mathrm{d} m}=0 .
$$

Now,

$$
\frac{\mathrm{dTP}_{v}(m)}{\mathrm{d} m}=\frac{1}{H(M)} \frac{\mathrm{d} J(m)}{\mathrm{d} m}-\frac{J(m)}{(H(M))^{2}} T_{b}
$$

where

$$
H(m)=T_{p}+m T_{b}>0
$$

and

$$
\begin{aligned}
J(m)= & \left\{v m D(1+L) \log \left[\frac{1+L}{1+L-T_{b}}\right]-A_{v}-c P(1+L)\left[1-e^{\frac{-m q}{P\left(1+L-T_{v}\right)}}\right]\right. \\
& -h_{v} P\left[\frac{\log (1+L)}{2}\left\{(1+L)^{2}-\left(1+L-T_{s}\right)^{2}\right\}\right] \\
& +h_{v} P\left[\frac{(1+L)^{2}}{2}\left\{\log (1+L)-\frac{1}{2}\right\}-\frac{1}{2}\left(1+L-T_{s}\right)^{2}\left\{\log \left(1+L-T_{s}\right)-\frac{1}{2}\right\}\right] \\
& -h_{v} \frac{m q}{\left(1+L-T_{v}\right)}\left[\left\{(1+L) T_{v}-\frac{T_{v}^{2}}{2}\right\}-\left\{(1+L) T_{s}-\frac{T_{s}^{2}}{2}\right\}\right] \\
& +h_{v} T_{b} \frac{m(m-1)}{2} D(1+L) \log \left[\frac{1+L}{1+L-T_{b}}\right] \\
& \left.-p_{c}\left[\left(m T_{v}-\frac{\left(1-e^{-\varphi m T_{v}}\right)}{\varphi}\right) \frac{\gamma p\left(p^{m}-1\right)}{p-1}+\frac{\left(1-e^{-\varphi m T_{v}}\right)}{\varphi} \frac{\gamma^{\prime}\left(p_{0}^{m}-1\right)}{p_{0}-1}\right]\right\} .
\end{aligned}
$$




\subsection{Centralized policy}

In the centralized policy, all players cooperatively decide the variables which maximize the whole system profit. The whole system's profit for three cases is given by

$$
\mathrm{TP}_{c}\left(m, T_{b}\right)=\mathrm{TP}_{b}\left(T_{b}\right)+\mathrm{TP}_{v}(m) .
$$

Proposition 4.3. The joint total profit function per unit time $\operatorname{TP}_{c}\left(m, T_{b}\right)$ is a strictly pseudo-concave function in $m$ and $T_{b}$, and hence attains its maxima for the optimal values of $T_{b}^{*}$ and $m^{*}$.

Proof. See Appendix C.

Equation (4.17) can be written as,

$$
\operatorname{TP}_{c}\left(m, T_{b}\right)=F\left(m, T_{b}\right)+D G\left(m, T_{b}\right)
$$

where

$$
\begin{aligned}
& F\left(m, T_{b}\right)=\frac{h_{v} P}{T_{v}+T_{b}}\left[\frac{(1+L)^{2}}{2}\left\{\log (1+L)-\frac{1}{2}\right\}-\frac{\left(1+L-T_{s}\right)^{2}}{2}\left\{\log \left(1+L-T_{s}\right)-\frac{1}{2}\right\}\right]-\frac{1}{T_{b}}\left(A_{b}+c_{T}\right) \\
& -\frac{1}{T_{v}+T_{b}}\left\{A_{v}+c P(1+L)\left[1-e^{\frac{-m q}{P\left(1+L-T_{v}\right)}}\right]+h_{v} P\left[\frac{\log (1+L)}{2}\left\{(1+L)^{2}-\left(1+L-T_{s}\right)^{2}\right\}\right]\right. \\
& -\frac{h_{v}}{T_{v}+T_{b}} \frac{m q}{\left(1+L-T_{v}\right)}\left[\left\{(1+L) T_{v}-\frac{T_{v}^{2}}{2}\right\}-\left\{(1+L) T_{s}-\frac{T_{s}^{2}}{2}\right\}\right] \\
& -\frac{p_{c}}{T_{v}+T_{b}}\left[\left(m T_{v}-\frac{\left(1-e^{-\varphi m T_{v}}\right)}{\varphi}\right) \frac{\gamma p\left(p^{m}-1\right)}{p-1}+\frac{\left(1-e^{-\varphi m T_{v}}\right)}{\varphi} \frac{\gamma^{\prime}\left(p_{0}^{m}-1\right)}{p_{0}-1}\right]
\end{aligned}
$$

and

$$
\begin{aligned}
G\left(m, T_{b}\right)= & s-\frac{1}{T_{b}}\left[\left(v+c_{t}\right)(1+L) \log \left(\frac{1+L}{1+L-T_{b}}\right)\right] \\
& -\frac{h_{b}}{T_{b}}\left[\frac{1}{2}\left(1+L^{2}\right) \log \left(\frac{1+L}{1+L-T_{b}}\right)+L \log \left(\frac{1+L}{1+L-T_{b}}\right)-\frac{1}{2} T_{b}-\frac{1}{2} L T_{b}+\frac{1}{4} T_{b}^{2}\right] \\
& +\frac{1}{T_{v}+T_{b}}\left\{v m(1+L) \log \left[\frac{1+L}{1+L-T_{b}}\right]+h_{v} T_{b} \frac{m(m-1)}{2}(1+L) \log \left[\frac{1+L}{1+L-T_{b}}\right]\right\} .
\end{aligned}
$$

Therefore, the crisp problem becomes

$$
\left\{\begin{array}{c}
\text { Maximize } Z=F+D G \\
\text { Subject to the conditions } T_{v}=T_{p}+(m-1) T_{b} .
\end{array}\right.
$$

\section{Formulation of FUZzy mathematical MODEL}

Here we assume the demand function varies with the variation of cycle time which carries some uncertainty, a part of learning theory. Thus, employing the theory of dense fuzzy set we may assume the demand function as a triangular dense fuzzy number whose membership function is stated below.

$$
\mu(D, n)= \begin{cases}0, & \text { if } D<d\left(1-\frac{\rho}{1+n}\right) \text { and } D>d\left(1+\frac{\sigma}{1+n}\right) \\ \frac{D-d\left(1-\frac{\rho}{1+n}\right)}{\frac{\rho d}{1+n}}, & \text { if } d\left(1-\frac{\rho}{1+n}\right) \leq D \leq d \\ \frac{d\left(1+\frac{\sigma}{1+n}\right)-D}{\frac{\sigma d}{1+n}}, & \text { if } d \leq D \leq d\left(1+\frac{\sigma}{1+n}\right) .\end{cases}
$$


Now, our fuzzy problem is given by

$$
\left\{\begin{array}{c}
\text { Maximize } \tilde{Z}=F+\tilde{D} G \\
\text { Subject to the conditions } T=T_{p}+(m-1) T_{b} .
\end{array}\right.
$$

From (4.14) we write,

$$
D=(Z-F) / G .
$$

Now, utilizing (5.3) in (5.2) we have the fuzzy membership function of the integrated profit function as

$$
\begin{gathered}
\mu(Z, n)= \begin{cases}0, & \text { if }(Z-F) / G<d\left(1-\frac{\rho}{1+n}\right) \text { and }(Z-F) / G>d\left(1+\frac{\sigma}{1+n}\right) \\
\frac{(Z-F) / G-d\left(1-\frac{\rho}{1+n}\right)}{\frac{\rho d}{1+n},}, & \text { if } d\left(1-\frac{\rho}{1+n}\right) \leq(Z-F) / G \leq d \\
\frac{d\left(1+\frac{\sigma}{1+n}\right)-(Z-F) / G}{\frac{\sigma d}{1+n}}, & \text { if } d \leq(Z-F) / G \leq d\left(1+\frac{\sigma}{1+n}\right)\end{cases} \\
\Rightarrow \mu(Z, n)= \begin{cases}0, & \text { if } Z<F+G d\left(1-\frac{\rho}{1+n}\right) \text { and } Z>F+G d\left(1+\frac{\sigma}{1+n}\right) \\
\frac{Z-F-G d\left(1-\frac{\rho}{1+n}\right)}{\frac{G \rho d}{1+n}}, & \text { if } F+G d\left(1-\frac{\rho}{1+n}\right) \leq Z \leq G d \\
\frac{F+G d\left(1+\frac{\sigma}{1+n}\right)-Z}{\frac{G \sigma d}{1+n}}, & \text { if } G d \leq Z \leq F+G d\left(1+\frac{\sigma}{1+n}\right) .\end{cases}
\end{gathered}
$$

Now, the $\alpha$-cut interval of $\mu(Z, T)$ is given by

$$
\left[Z_{L}, Z_{R}\right]=\left[F+G d\left(1+\frac{\sigma}{1+n}\right)-\alpha \frac{G \sigma d}{1+n}, F+G d\left(1-\frac{\rho}{1+n}\right)+\alpha \frac{G \rho d}{1+n}\right] .
$$

Therefore, as per De and Mahata [7], the defuzzified index value of the fuzzy objective function is given by

$$
\begin{aligned}
I(Z) & =\frac{1}{2 N} \sum_{n=0}^{N-1} \int_{0}^{1}\left[F+G d\left(1+\frac{\sigma}{1+n}\right)-\alpha \frac{G \sigma d}{1+n}+F+G d\left(1-\frac{\rho}{1+n}\right)+\alpha \frac{G \rho d}{1+n}\right] \mathrm{d} \alpha \\
& =\frac{1}{2 N} \sum_{n=0}^{N-1}\left[2 F+G d\left\{2+\frac{\sigma-\rho}{1+n}+\frac{(\rho-\sigma)}{2(1+n)}\right\}\right] \\
& =F+G d+\frac{1}{4 N} \sum_{n=0}^{N-1}\left[\frac{\sigma-\rho}{1+n}\right]=F+G d+\frac{(\sigma-\rho)}{4 N}\left[1+\frac{1}{2}+\frac{1}{3}+\frac{1}{4}+\ldots+\frac{1}{N}\right]
\end{aligned}
$$

where the functions $F$ and $G$ are obtained from (4.18).

Then, the equivalent crisp problem of the fuzzy problem is

$$
\left\{\begin{array}{c}
\text { Maximize } I(Z)=F+G d+\frac{(\sigma-\rho)}{4 N}\left[1+\frac{1}{2}+\frac{1}{3}+\frac{1}{4}+\ldots+\frac{1}{N}\right] \\
\text { Subject to } I(q)=(1+L) d \log \left[\frac{1+L}{1+L-T_{b}}\right]\left[1+\frac{\sigma-\rho}{4 N}\left\{1+\frac{1}{2}+\frac{1}{3}+\ldots+\frac{1}{N}\right\}\right]
\end{array}\right.
$$

where $q$ is obtained from equation (4.5) and we get

$$
\begin{aligned}
q & =I_{b}(0)=D(1+L) \log \left[\frac{1+L}{1+L-T_{b}}\right] \\
\Rightarrow I(q) & =\frac{(1+L) d}{2 N} \log \left[\frac{1+L}{1+L-T_{b}}\right]\left[2 N+\frac{\sigma-\rho}{2}\left\{\frac{1}{1+0}+\frac{1}{1+1}+\frac{1}{1+2}+\ldots+\frac{1}{1+N}\right\}\right] \\
& =(1+L) d \log \left[\frac{1+L}{1+L-T_{b}}\right]\left[1+\frac{\sigma-\rho}{4 N}\left\{1+\frac{1}{2}+\frac{1}{3}+\ldots+\frac{1}{N}\right\}\right], \quad \text { for } N=1,2,3, \ldots
\end{aligned}
$$


TABLE 1. Optimal results for the proposed models.

\begin{tabular}{llllllll}
\hline \hline Model & $\begin{array}{l}T_{b}^{*} \\
(\text { months }\end{array}$ & $\begin{array}{l}T_{v}^{*} \\
\text { (months) }\end{array}$ & $\begin{array}{l}q^{*} \\
\text { (units) }\end{array}$ & $n^{*} \begin{array}{l}\mathrm{TP}_{b}^{*} \\
(\$)\end{array}$ & $\begin{array}{l}\mathrm{TP}_{v}^{*} \\
(\$)\end{array}$ & $\begin{array}{l}\mathrm{TP}_{c}^{*} \\
(\$)\end{array}$ \\
\hline Crisp Model (decentralized) Buyer & 0.140 & & 358.521 & 3 & 38281.17 & \\
\hline Crisp Model (decentralized) Vendor & & 0.3644 & & 3 & & 26349.24 & 54630.41 \\
\hline Crisp Model (Centralized) & 0.0980 & 0.3152 & 249.083 & 4 & 37999.21 & 27010.70 & 65009.91 \\
\hline Fuzzy Model (Centralized) & 0.0964 & 0.3159 & 249.92 & 4 & 38794.58 & 27549.47 & 66344.05 \\
\hline
\end{tabular}

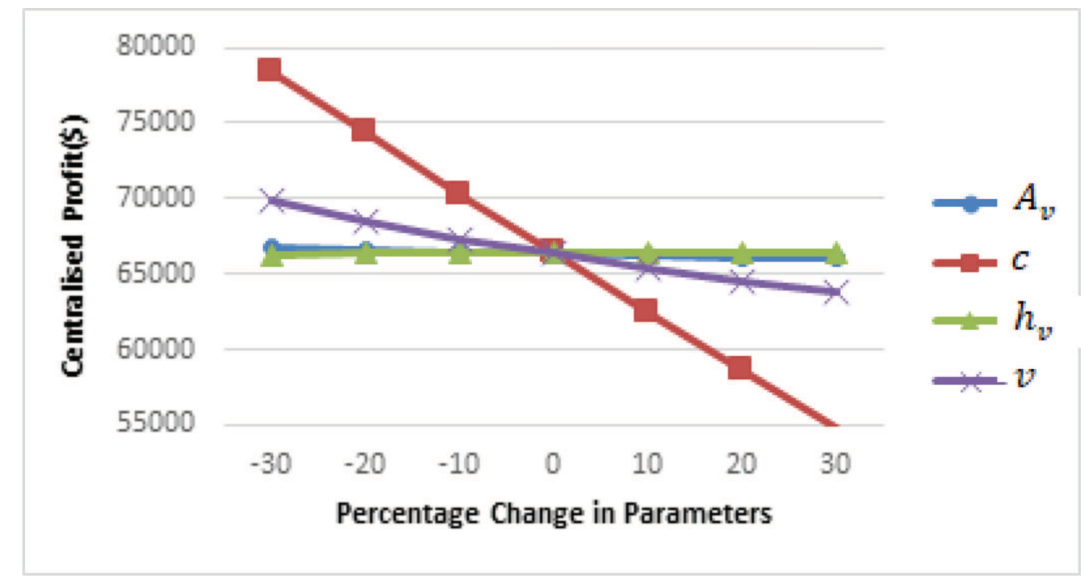

Figure 4. Centralized profit with respect to \% change in $A_{v}, c, h_{v}$ and $v$.

\section{NumericAl EXAMPLE}

To illustrate the numerical study, we consider the following initial dataset: Let, $D=1500 \mathrm{unit} / \mathrm{month}$, $P=5000$ units/month, $A_{b}=\$ 250 /$ order, $A_{v}=\$ 500 /$ setup, $c=\$ 16 /$ unit, $s=\$ 50 /$ unit, $v=\$ 30 /$ unit, $h_{b}=\$ 0.5 /$ unit $/$ month, $h_{v}=\$ 0.3 /$ unit $/$ month, $c_{T}=\$ 50 /$ ship, $c_{t}=\$ 3 /$ unit, $L=6$ month; scale parameter of current pollution $\gamma=10$, scale parameter of initial pollution $\gamma^{\prime}=5$, pollution absorbance rate by the nature $\varphi=0.01$, threshold amount of pollution rate $p_{0}=1.5$, rate of current pollution input $p=2.5$, unit pollution cost $p_{c}=\$ 0.2$. Taking with the help of LINGO software, the following optimal results are found.

Table 1 gives the optimum profit values of the proposed model under crisp (centralized and decentralized cases) and fuzzy centralized model respectively. It is seen that, for decentralized crisp model the total profit earned by the buyer and the vendor is $\$ 54630.41$ but that for centralized model it becomes $\$ 65009.91$ which is almost $19 \%$ higher than earlier value. However, the dense fuzzy model for 4 consecutive learning cycle times the profit of the chain is raised to $\$ 66344.05$ which is almost $2.01 \%$ extra gain of the decision maker. In this case, the order quantity assumes value 249.92 units with respect to the buyer's cycle time 0.1 month and that for vendor it becomes 0.32 month respectively.

\subsection{Graphical illustrations on sensitivity analysis}

In this subsection, we now carry out the effects of changes in the key system parameters on the optimal solution of the proposed supply chain model. We take a sensitivity analysis by changing the value of each parameter on and from $(-30 \%,-20 \%,-10 \%, 0 \%,+10 \%,+20,+30 \%)$ keeping the others unchanged. Based on the behavioral changes as reflected in Figures 4-7, we derive the following managerial insights. 


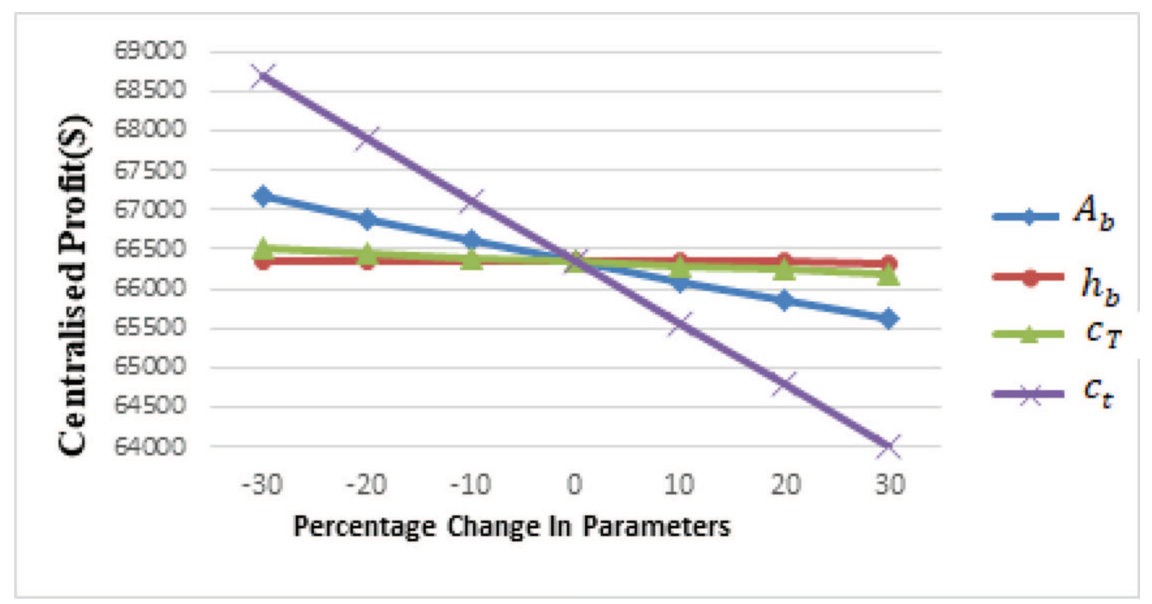

Figure 5. Centralized profit with respect to $\%$ change in $A_{b}, h_{b}, c_{T}$ and $c_{t}$.

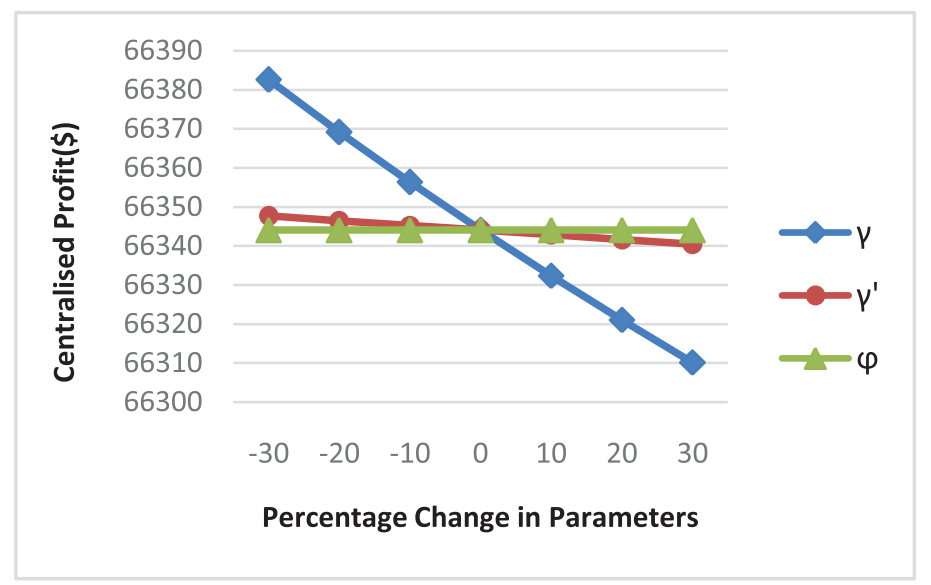

Figure 6. Centralized profit with respect to $\%$ change in $\gamma, \gamma^{\prime}$ and $\phi$.

Table 2 expresses the sensitivity analysis of the objective function over the parameters $A_{v}, c, h_{v}$, and $v$ for the changes on and from $(-30 \%,-20 \%,-10 \%)$ to $(+10 \%,+20 \%$ and $+30 \%)$ respectively. It is seen that, the vendor's unit production cost $(c)$ is highly responsible for average sensitivity of the joint profit function of centralized model and the other parameters like vendor's setup cost $\left(A_{v}\right)$, holding cost of the vendor and the vendor's unit supply price $v$ are less sensitive for similar types of changes. All parameters give profit enhancement of the model under learning frequency 4 in most of the cases.

The graphical representations are shown in Figure 4. The range of profit variation lies within $\$ 65000-70000$ for the change of parameters $A_{v}, h_{v}$, and $v$ only but for $c$ it lies beyond this limit.

Table 3 gives a sensitivity analysis for the parameters $A_{b}, h_{b}, c_{T}, c_{t}$ changing on and from $(-30 \%,-20 \%$, $-10 \%),(+10 \%,+20 \%$ and $+30 \%)$ respectively. We notice that all changes are poorly sensitive under fuzzy learning frequency 4 . It is also observed that

(a) When the buyer's fixed costs (such as the ordering cost or fixed shipping cost) increase, the buyer's order quantity and the vendor's order quantity increase. In addition, the buyer's total profit decreases because of 


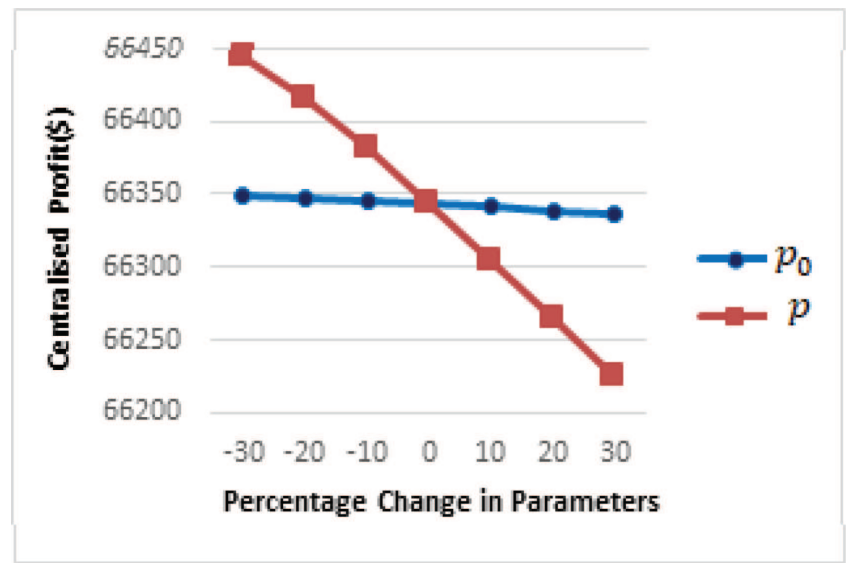

Figure 7 . Centralized profit with respect to $\%$ change in $p_{0}$ and $p$.

TABle 2. Sensitivity analysis for the parameters $A_{v}, c, h_{v}, v$.

\begin{tabular}{|c|c|c|c|c|c|c|c|c|c|}
\hline Parameter & $\begin{array}{l}\% \\
\text { change }\end{array}$ & $\begin{array}{l}T_{b}^{*} \\
\text { (months) }\end{array}$ & $\begin{array}{l}T_{v}^{*} \\
\text { (months) }\end{array}$ & $\begin{array}{l}q^{*} \\
\text { (units) }\end{array}$ & $n^{*}$ & $\begin{array}{l}\mathrm{TP}_{b}^{*} \\
(\$)\end{array}$ & $\begin{array}{l}\mathrm{TP}_{v}^{*} \\
(\$)\end{array}$ & $\begin{array}{l}\mathrm{TP}_{c}^{*} \\
(\$)\end{array}$ & $\begin{array}{l}\% \text { change } \\
\text { in } \mathrm{TP}_{c}^{*}\end{array}$ \\
\hline \multirow{7}{*}{$A_{v}$} & $-30 \%$ & 0.09300 & 0.30169 & 240.91 & 4 & 38732.44 & 27983.32 & 66715.77 & +0.56 \\
\hline & $-20 \%$ & 0.09416 & 0.30653 & 243.97 & 4 & 38754.44 & 27835.59 & 66590.04 & +0.37 \\
\hline & $-10 \%$ & 0.09530 & 0.31127 & 246.97 & 4 & 38775.12 & 27691.04 & 66466.17 & +0.18 \\
\hline & $0 \%$ & 0.09642 & 0.31590 & 249.92 & 4 & 38794.54 & 27549.47 & 66344.05 & 0 \\
\hline & $+10 \%$ & 0.09752 & 0.32043 & 252.83 & 4 & 38812.90 & 27410.71 & 66223.61 & -0.18 \\
\hline & $+20 \%$ & 0.09861 & 0.32488 & 255.70 & 4 & 38830.16 & 27274.60 & 66104.78 & -0.36 \\
\hline & $+30 \%$ & 0.09968 & 0.32925 & 258.52 & 4 & 38846.45 & 27141.00 & 65987.47 & -0.54 \\
\hline \multirow{7}{*}{$c$} & $-30 \%$ & 0.10082 & 0.38594 & 261.52 & 4 & 38862.95 & 39484.71 & 78347.67 & $\begin{array}{l}+18.09 \\
\end{array}$ \\
\hline & $-20 \%$ & 0.09826 & 0.36099 & 254.76 & 4 & 38824.59 & 35476.38 & 74300.98 & +11.99 \\
\hline & $-10 \%$ & 0.09677 & 0.33798 & 250.85 & 4 & 38800.52 & 31498.33 & 70298.87 & +5.96 \\
\hline & $0 \%$ & 0.09642 & 0.31590 & 249.92 & 4 & 38794.54 & 27549.47 & 66344.05 & 0 \\
\hline & $+10 \%$ & 0.09737 & 0.29380 & 252.42 & 3 & 38810.37 & 23632.15 & 62442.53 & -5.88 \\
\hline & $+20 \%$ & 0.09992 & 0.27081 & 259.15 & 3 & 38849.96 & 19754.38 & 58604.36 & -11.67 \\
\hline & $+30 \%$ & 0.10451 & 0.24651 & 271.24 & 3 & 38911.29 & 15933.29 & 54844.59 & -17.33 \\
\hline \multirow{7}{*}{$h_{v}$} & $-30 \%$ & & & No fe & sible & solution & & & \\
\hline & $-20 \%$ & 0.09651 & 0.31641 & 250.17 & 4 & 38796.17 & 27560.14 & 66356.33 & +0.02 \\
\hline & $-10 \%$ & 0.09647 & 0.31615 & 250.05 & 4 & 38795.37 & 27554.80 & 66350.19 & +0.01 \\
\hline & $0 \%$ & 0.09642 & 0.31590 & 249.92 & 4 & 38794.58 & 27549.47 & 66344.05 & 0 \\
\hline & $+10 \%$ & 0.09637 & 0.31564 & 249.80 & 4 & 38793.78 & 27544.13 & 66337.92 & -0.01 \\
\hline & $+20 \%$ & 0.09633 & 0.31538 & 249.68 & 4 & 38792.98 & 27538.81 & 66331.80 & -0.02 \\
\hline & $+30 \%$ & 0.09628 & 0.31513 & 249.55 & 4 & 38792.19 & 27533.48 & 66325.68 & -0.03 \\
\hline \multirow{7}{*}{$v$} & $-30 \%$ & 0.14269 & 0.23217 & 372.82 & 2 & 62601.81 & 7245.942 & 69847.76 & +5.28 \\
\hline & $-20 \%$ & 0.11731 & 0.27550 & 305.30 & 3 & 54635.10 & 13832.75 & 68467.87 & +3.20 \\
\hline & $-10 \%$ & 0.10415 & 0.30071 & 270.32 & 3 & 46693.00 & 20646.76 & 67339.78 & +1.50 \\
\hline & $0 \%$ & 0.09642 & 0.31590 & 249.92 & 4 & 38794.54 & 27549.47 & 66344.05 & 0 \\
\hline & $+10 \%$ & 0.09138 & 0.32557 & 236.64 & 4 & 30931.00 & 34495.39 & 65426.40 & -1.38 \\
\hline & $+20 \%$ & 0.08778 & 0.33209 & 227.18 & 4 & 23091.92 & 41467.83 & 64559.76 & -2.69 \\
\hline & $+30 \%$ & 0.0850 & 0.33669 & 219.97 & 4 & 15270.12 & 48459.20 & 63729.33 & -3.94 \\
\hline
\end{tabular}


TABLE 3. Sensitivity analysis of $A_{b}, h_{b}, c_{T}, c_{t}$.

\begin{tabular}{|c|c|c|c|c|c|c|c|c|c|}
\hline Parameter & $\begin{array}{l}\% \\
\text { change }\end{array}$ & $\begin{array}{l}T_{b}^{*} \\
\text { (months) }\end{array}$ & $\begin{array}{l}T_{v}^{*} \\
\text { (months) }\end{array}$ & $\begin{array}{l}q^{*} \\
\text { (units) }\end{array}$ & $n^{*}$ & $\begin{array}{l}\mathrm{TP}_{b}^{*} \\
(\$)\end{array}$ & $\begin{array}{l}\mathrm{TP}_{v}^{*} \\
(\$)\end{array}$ & $\begin{array}{l}\mathrm{TP}_{c}^{*} \\
(\$)\end{array}$ & $\begin{array}{l}\% \text { change } \\
\text { in } \mathrm{TP}_{c}^{*}\end{array}$ \\
\hline \multirow[t]{7}{*}{$A_{b}$} & $-30 \%$ & 0.08441 & 0.29457 & 218.34 & 4 & 39423.65 & 27450.47 & 67174.13 & +1.25 \\
\hline & $-20 \%$ & 0.08850 & 0.30227 & 229.09 & 4 & 39202.10 & 27682.84 & 66884.96 & +0.82 \\
\hline & $-10 \%$ & 0.09250 & 0.30935 & 239.61 & 4 & 38992.95 & 27615.75 & 66608.72 & +0.40 \\
\hline & $0 \%$ & 0.09642 & 0.31590 & 249.92 & 4 & 38794.54 & 27549.47 & 66344.05 & 0 \\
\hline & $+10 \%$ & 0.10026 & 0.32196 & 260.05 & 4 & 38605.63 & 27484.19 & 66089.83 & -0.38 \\
\hline & $+20 \%$ & 0.10404 & 0.32759 & 270.02 & 4 & 38425.03 & 27420.05 & 65845.09 & -0.75 \\
\hline & $+30 \%$ & 0.10775 & 0.33288 & 279.82 & 3 & 38251.84 & 27357.15 & 65609.00 & -1.11 \\
\hline \multirow[t]{7}{*}{$h_{b}$} & $-30 \%$ & 0.09670 & 0.31635 & 250.67 & 4 & 38818.06 & 27544.65 & 66362.72 & +0.03 \\
\hline & $-20 \%$ & 0.09661 & 0.31621 & 250.42 & 4 & 38810.22 & 27546.26 & 66356.49 & +0.02 \\
\hline & $-10 \%$ & 0.09651 & 0.31605 & 250.17 & 4 & 38802.39 & 27547.87 & 66350.27 & +0.01 \\
\hline & $0 \%$ & 0.09642 & 0.31590 & 249.92 & 4 & 38794.58 & 27549.47 & 66344.05 & 0 \\
\hline & $+10 \%$ & 0.09633 & 0.31575 & 249.68 & 4 & 38786.77 & 27551.06 & 66337.84 & -0.01 \\
\hline & $+20 \%$ & 0.09263 & 0.31560 & 249.43 & 4 & 38778.98 & 27552.64 & 66331.64 & -0.02 \\
\hline & $+30 \%$ & 0.09144 & 0.31544 & 249.18 & 4 & 38771.20 & 27554.22 & 66325.44 & -0.03 \\
\hline \multirow[t]{7}{*}{$c_{T}$} & $-30 \%$ & 0.09408 & 0.31204 & 243.76 & 4 & 38912.39 & 27589.13 & 66501.53 & +0.24 \\
\hline & $-20 \%$ & 0.09486 & 0.31334 & 245.82 & 4 & 38872.72 & 27575.87 & 66448.61 & +0.16 \\
\hline & $-10 \%$ & 0.09564 & 0.31463 & 247.87 & 4 & 38833.46 & 27562.65 & 66396.12 & +0.08 \\
\hline & $0 \%$ & 0.09642 & 0.31590 & 249.92 & 4 & 38794.58 & 27549.47 & 66344.05 & 0 \\
\hline & $+10 \%$ & 0.09719 & 0.31715 & 251.96 & 4 & 38756.07 & 27536.32 & 66292.41 & -0.08 \\
\hline & $+20 \%$ & 0.09797 & 0.31838 & 253.99 & 4 & 38717.93 & 27523.23 & 66241.17 & -0.16 \\
\hline & $+30 \%$ & 0.09873 & 0.31959 & 256.02 & 4 & 38680.15 & 27510.17 & 66190.33 & -0.23 \\
\hline \multirow[t]{7}{*}{$c_{t}$} & $-30 \%$ & 0.09700 & 0.31684 & 251.46 & 4 & 41137.27 & 27539.58 & 68676.86 & +3.52 \\
\hline & $-20 \%$ & 0.09681 & 0.31653 & 250.94 & 4 & 40356.32 & 27542.90 & 67899.23 & +2.34 \\
\hline & $-10 \%$ & 0.09661 & 0.31621 & 250.43 & 4 & 39575.42 & 27546.19 & 67121.63 & +1.17 \\
\hline & $0 \%$ & 0.09642 & 0.31590 & 249.92 & 4 & 38794.58 & 27549.47 & 66344.05 & 0 \\
\hline & $+10 \%$ & 0.09623 & 0.31559 & 249.42 & 4 & 38013.78 & 27552.71 & 65566.50 & -1.17 \\
\hline & $+20 \%$ & 0.09604 & 0.31528 & 248.92 & 4 & 37233.03 & 27555.94 & 64788.98 & -2.34 \\
\hline & $+30 \%$ & 0.09585 & 0.31497 & 248.42 & 4 & 36452.33 & 27559.14 & 64011.48 & -3.52 \\
\hline
\end{tabular}

the increased fixed costs, whereas the vendor's profits increase due to the buyer's increased order quantity, but in the whole system, profit value decreases slightly as shown in Figure 5.

(b) As the buyer's holding cost or variable shipping cost increases, the vendor's optimal shipping quantity, and the buyer's optimal order quantity decrease such that the total system profit decreases slightly (shown in Fig. 5).

Table 4 discusses the effect of sensitivity of pollution parameters $\gamma, \gamma^{\prime}$ and $\phi$ upon the centralized model whenever we perform the changes on and from $(-30 \%,-20 \%,-10 \%),(+10 \%,+20 \%$ and $+30 \%)$ respectively. It is seen that the parameters $\gamma, \gamma^{\prime}$ have negligible but unidirectional change that may contribute extra profit to the centralized model and the parameter $\phi$ gives almost no profit enhancement of the model. The graphical illustrations of this analysis in shown in Figure 6. From this figure we see that the parameter $\gamma$ has a significant contribution that ranges the profit value within $\$ 66310-66380$, but for the others it is limited to $\$ 66340-\$ 66350$ exclusively.

Table 5 reveals the sensitivity of the pollution parameters $p_{0}$ and $p$ subject to the changes on and from $(-30 \%,-20 \%,-10 \%),(+10 \%,+20 \%$ and $+30 \%)$ respectively. All changes have opposite directional but of low sensitivity. The graphical illustration shows that (Fig. 7), the parameter $p$ has high fluctuating range $\$ 66200-$ 66450 comparative to the other parameter $p_{0}$ that creates profit value near $\$ 66350$ explicitly. 
TABLE 4. Sensitivity analysis of the pollution parameters $\gamma, \gamma^{\prime}$ and $\phi$.

\begin{tabular}{llllllllll}
\hline \hline Parameter & $\begin{array}{l}\% \\
\text { change }\end{array}$ & $\begin{array}{l}T_{b}^{*} \\
\text { (months) }\end{array}$ & $\begin{array}{l}T_{v}^{*} \\
\text { (months) }\end{array}$ & $\begin{array}{l}q^{*} \\
\text { (units) }\end{array}$ & $n^{*}$ & $\begin{array}{l}\mathrm{TP}_{b}^{*} \\
(\$)\end{array}$ & $\begin{array}{l}\mathrm{TP}_{v}^{*} \\
(\$)\end{array}$ & $\begin{array}{l}\mathrm{TP}_{c}^{*} \\
(\$)\end{array}$ & $\begin{array}{l}\text { \% change } \\
\text { in TP }\end{array}$ \\
\hline & $-30 \%$ & 0.09515 & 0.32044 & 246.58 & 4 & 38772.50 & 27610.05 & 66382.56 & +0.06 \\
& $-20 \%$ & 0.09561 & 0.31880 & 247.78 & 4 & 38780.58 & 27588.51 & 66369.10 & +0.04 \\
& $-10 \%$ & 0.09603 & 0.31730 & 248.89 & 4 & 38787.89 & 27568.39 & 66356.29 & +0.02 \\
& $0 \%$ & 0.09642 & 0.31590 & 249.92 & 4 & 38794.58 & 27549.47 & 66344.05 & 0 \\
& $+10 \%$ & 0.09679 & 0.31459 & 250.88 & 4 & 38800.72 & 27531.58 & 66332.32 & -0.02 \\
& $+20 \%$ & 0.09713 & 0.31337 & 251.79 & 4 & 38806.42 & 27514.61 & 66321.04 & -0.03 \\
& $+30 \%$ & 0.09745 & 0.31221 & 252.64 & 4 & 38811.71 & 27498.45 & 66310.17 & -0.05 \\
\hline & $-30 \%$ & 0.09634 & 0.31598 & 249.72 & 4 & 38793.24 & 27554.43 & 66347.68 & +0.01 \\
$\gamma^{\prime}$ & $-20 \%$ & 0.09637 & 0.31595 & 249.78 & 4 & 38793.68 & 27552.77 & 66346.47 & +0.00 \\
& $-10 \%$ & 0.09639 & 0.31593 & 249.85 & 4 & 38794.13 & 27551.12 & 66345.26 & +0.00 \\
& $0 \%$ & 0.09642 & 0.31590 & 249.92 & 4 & 38794.58 & 27549.47 & 66344.05 & 0 \\
& $+10 \%$ & 0.09645 & 0.31587 & 249.99 & 4 & 38795.02 & 27547.82 & 66342.85 & -0.00 \\
& $+20 \%$ & 0.09647 & 0.31585 & 250.06 & 4 & 38795.46 & 27546.17 & 66341.64 & -0.00 \\
& $+30 \%$ & 0.09650 & 0.31582 & 250.13 & 4 & 38795.90 & 27544.52 & 66340.44 & -0.01 \\
\hline & $-30 \%$ & 0.09642 & 0.31590 & 249.92 & 4 & 38794.58 & 27549.47 & 66344.05 & 0 \\
& $-20 \%$ & 0.09642 & 0.31590 & 249.92 & 4 & 38794.58 & 27549.47 & 66344.05 & 0 \\
& $-10 \%$ & 0.09642 & 0.31590 & 249.92 & 4 & 38794.58 & 27549.47 & 66344.05 & 0 \\
& $0 \%$ & 0.09642 & 0.31590 & 249.92 & 4 & 38794.58 & 27549.47 & 66344.05 & 0 \\
& $+10 \%$ & 0.09642 & 0.31590 & 249.92 & 4 & 38794.58 & 27549.47 & 66344.05 & 0 \\
& $+20 \%$ & 0.09642 & 0.31590 & 249.92 & 4 & 38794.58 & 27549.47 & 66344.05 & 0 \\
& $+30 \%$ & 0.09642 & 0.31590 & 249.92 & 4 & 38794.58 & 27549.47 & 66344.05 & 0 \\
\hline
\end{tabular}

TABLE 5. Sensitivity analysis of $p_{0}$ and $p$.

\begin{tabular}{llllllllll}
\hline \hline Parameter & $\begin{array}{l}\% \\
\text { change }\end{array}$ & $\begin{array}{l}T_{b}^{*} \\
\text { (months) }\end{array}$ & $\begin{array}{l}T_{v}^{*} \\
(\text { months })\end{array}$ & $\begin{array}{l}q^{*} \\
\text { (units) }\end{array}$ & $n^{*}$ & $\begin{array}{l}\mathrm{TP}_{b}^{*} \\
(\$)\end{array}$ & $\begin{array}{l}\mathrm{TP}_{v}^{*} \\
(\$)\end{array}$ & $\begin{array}{l}\mathrm{TP}_{c}^{*} \\
(\$)\end{array}$ & $\begin{array}{l}\text { \% change } \\
\text { in TP }\end{array}$ \\
\hline & $-30 \%$ & 0.09627 & 0.31611 & 249.52 & 4 & 38791.95 & 27557.40 & 66349.36 & +0.0080 \\
& $-20 \%$ & 0.09631 & 0.31605 & 249.62 & 4 & 38792.64 & 27555.20 & 66347.85 & +0.0057 \\
$p_{0}$ & $-10 \%$ & 0.09636 & 0.31599 & 249.76 & 4 & 38793.51 & 27552.57 & 66346.09 & +0.0031 \\
& $0 \%$ & 0.09642 & 0.31590 & 249.92 & 4 & 38794.58 & 27549.47 & 66344.05 & 0 \\
& $+10 \%$ & 0.09650 & 0.31579 & 250.12 & 4 & 38795.86 & 27545.86 & 66341.74 & -0.0035 \\
& $+20 \%$ & 0.09659 & 0.31565 & 250.36 & 4 & 38797.39 & 27541.72 & 66339.13 & -0.0074 \\
& $+30 \%$ & 0.09669 & 0.31549 & 250.64 & 4 & 38799.17 & 27537.03 & 66336.21 & -0.01181 \\
\hline & $-30 \%$ & 0.09220 & 0.33047 & 238.80 & 4 & 38716.59 & 27728.00 & 66444.60 & +0.1516 \\
& $-20 \%$ & 0.09349 & 0.32588 & 242.22 & 4 & 38741.96 & 27673.34 & 66415.31 & +0.1074 \\
& $-10 \%$ & 0.09493 & 0.32092 & 246.01 & 4 & 38768.61 & 27612.70 & 66381.32 & +0.0562 \\
& $0 \%$ & 0.09642 & 0.31590 & 249.92 & 4 & 38794.58 & 27549.47 & 66344.05 & 0 \\
& $+10 \%$ & 0.09789 & 0.31103 & 253.79 & 4 & 38818.77 & 27486.00 & 66304.79 & -0.0592 \\
& $+20 \%$ & 0.09931 & 0.30642 & 257.53 & 3 & 38840.79 & 27423.72 & 66264.52 & -0.1199 \\
& $+30 \%$ & 0.10065 & 0.30209 & 261.08 & 3 & 38860.58 & 27363.40 & 66223.99 & -0.1801 \\
\hline
\end{tabular}


TABLE 6 . Sensitivity analysis of $P$ and $D$.

\begin{tabular}{lllllllllll}
\hline \hline Parameter & $\begin{array}{l}\% \\
\text { change }\end{array}$ & $\begin{array}{l}T_{b}^{*} \\
\text { (months) }\end{array}$ & $\begin{array}{l}T_{v}^{*} \\
(\text { months })\end{array}$ & $\begin{array}{l}q^{*} \\
\text { (units) }\end{array}$ & $n^{*}$ & $\begin{array}{l}\mathrm{TP}_{b}^{*} \\
(\$)\end{array}$ & $\begin{array}{l}\mathrm{TP}_{v}^{*} \\
(\$)\end{array}$ & $\begin{array}{l}\mathrm{TP}_{c}^{*} \\
(\$)\end{array}$ & $\begin{array}{l}\text { \% change } \\
\text { in TP }\end{array}$ \\
\hline & $-30 \%$ & & & \multicolumn{6}{c}{ No feasible solution } \\
& $-20 \%$ & 0.09381 & 0.34938 & 243.04 & 4 & 38747.88 & 26968.42 & 65716.31 & -0.95 \\
& $-10 \%$ & 0.09493 & 0.33121 & 245.99 & 4 & 38768.48 & 27282.47 & 66050.96 & -0.44 \\
& $0 \%$ & 0.09642 & 0.31590 & 249.92 & 4 & 38794.54 & 27549.47 & 66344.05 & 0 \\
& $+10 \%$ & 0.09818 & 0.30259 & 254.56 & 4 & 38823.41 & 27781.08 & 66604.50 & +0.39 \\
& $+20 \%$ & 0.10013 & 0.29078 & 259.72 & 3 & 38853.10 & 27985.61 & 66838.72 & +0.75 \\
& $+30 \%$ & 0.10223 & 0.28013 & 256.25 & 3 & 38882.37 & 28169.05 & 67051.44 & +1.07 \\
\hline & $-30 \%$ & 0.13375 & 0.29687 & 244.23 & 3 & 26687.94 & 19280.38 & 45968.33 & -30.71 \\
& $-20 \%$ & 0.11734 & 0.30568 & 244.18 & 3 & 30710.76 & 22037.66 & 52748.43 & -20.49 \\
& $-10 \%$ & 0.10538 & 0.31174 & 246.22 & 3 & 34744.92 & 24797.18 & 59542.12 & -10.25 \\
& $0 \%$ & 0.09642 & 0.31590 & 249.92 & 4 & 38794.58 & 27549.47 & 66344.05 & 0 \\
& $+10 \%$ & 0.0895 & 0.31880 & 254.92 & 4 & 42861.16 & 30288.42 & 73149.60 & +10.26 \\
& $+20 \%$ & 0.08406 & 0.32091 & 260.90 & 4 & 46944.48 & 33010.46 & 79954.95 & +20.52 \\
& $+30 \%$ & 0.07965 & 0.32257 & 267.62 & 4 & 51043.56 & 35713.58 & 86757.15 & +30.77 \\
\hline
\end{tabular}

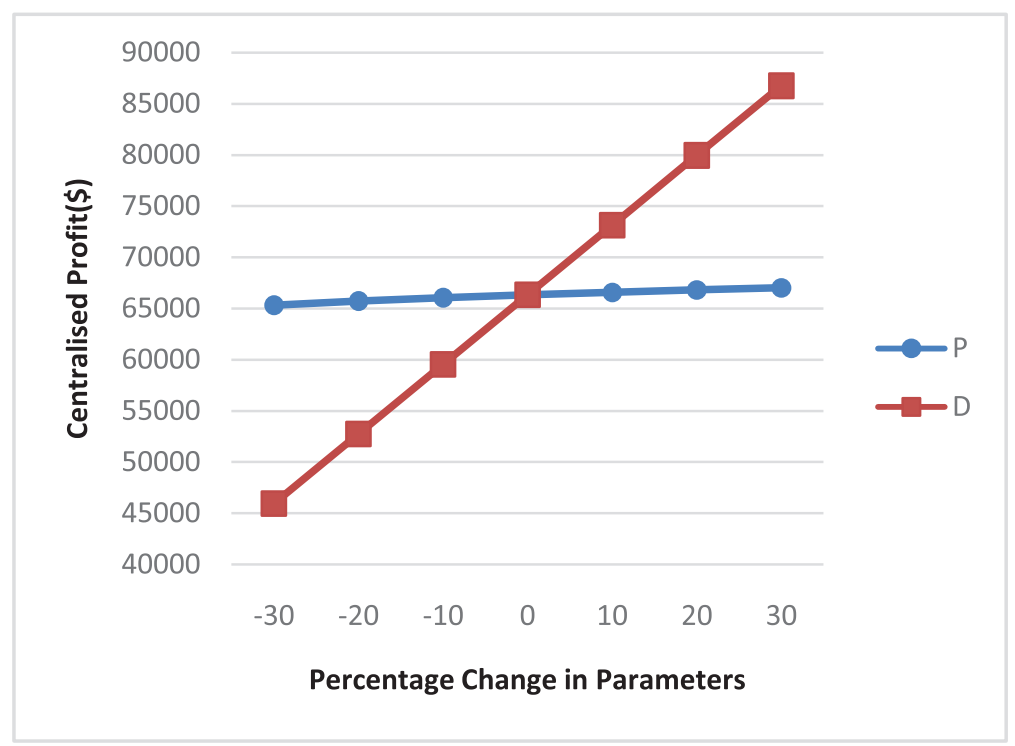

Figure 8 . Centralized profit with respect to $\%$ change in $P$ and $D$.

Table 6 gives a sensitivity analysis for the vendor's production rate $P$ and the demand rate $D$ subject to the changes on and from $(-30 \%,-20 \%,-10 \%, 0 \%,+10 \%,+20 \%$ and $+30 \%)$ simultaneously. It is seen that; the demand parameter has an average unidirectional sensitivity upon the change of profit function but the production rate has no significant sensitivity. The whole table shows that the order quantity keeps a range 243.04-267.62 units and the respective cycle time range becomes nearly $0.29-0.35$ month with respect to the learning parametric range 3-4. The graphical illustration for this analysis is given in Figure 8 which reveals that, when the market demand increases, the vendor's shipping quantity as well as the buyer's order quantity increases, which increases the whole system profit. With the increased productivity of the vendor, the vendor's optimal shipping quantity, and the buyer's optimal order quantity increase. The profit range assumes value 
TABLE 7. Sensitivity analysis of $L$.

\begin{tabular}{llllllllll}
\hline \hline Parameter & $\begin{array}{l}\% \\
\text { change }\end{array}$ & $\begin{array}{l}T_{b}^{*} \\
\text { (months) }\end{array}$ & $\begin{array}{l}T_{v}^{*} \\
\text { (months) }\end{array}$ & $\begin{array}{l}q^{*} \\
\text { (units) }\end{array}$ & $n^{*}$ & $\begin{array}{l}\mathrm{TP}_{b}^{*} \\
(\$)\end{array}$ & $\begin{array}{l}\mathrm{TP}_{v}^{*} \\
(\$)\end{array}$ & $\begin{array}{l}\mathrm{TP}_{c}^{*} \\
(\$)\end{array}$ & $\begin{array}{l}\text { \% change } \\
\text { in TP }\end{array}$ \\
\hline \multirow{4}{*}{$L$} & $-30 \%$ & 0.08809 & 0.27667 & 228.88 & 4 & 38304.51 & 27066.05 & 65370.57 & -1.47 \\
& $-20 \%$ & 0.09102 & 0.29101 & 235.72 & 4 & 38485.21 & 27210.52 & 65659.73 & -0.98 \\
& $-10 \%$ & 0.09373 & 0.30340 & 243.11 & 4 & 38648.24 & 27406.22 & 66054.47 & -0.45 \\
& $0 \%$ & 0.09642 & 0.31590 & 249.92 & 4 & 38794.58 & 27549.47 & 66344.05 & 0 \\
& $+10 \%$ & 0.09904 & 0.32789 & 256.55 & 4 & 38297.41 & 27678.58 & 66606.00 & +0.39 \\
& $+20 \%$ & 0.10158 & 0.33942 & 262.99 & 4 & 39048.70 & 27795.67 & 66844.38 & +0.75 \\
& $+30 \%$ & 0.10406 & 0.35054 & 269.28 & 4 & 39160.01 & 27902.45 & 67062.47 & +1.08 \\
\hline
\end{tabular}

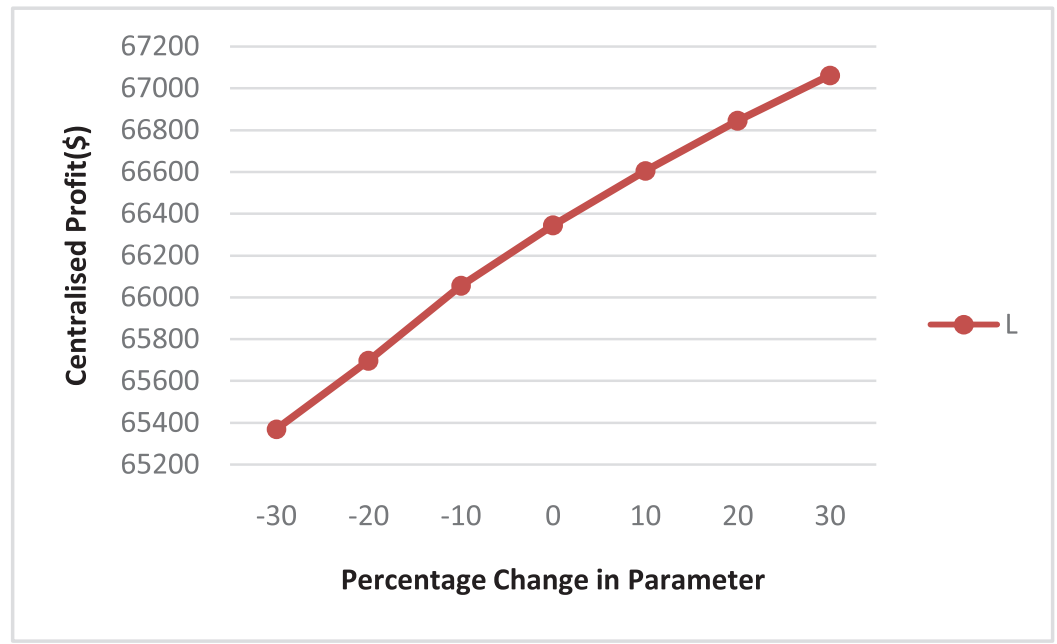

Figure 9. Centralized profit with respect to \% change in $L$.

$\$ 45000-\$ 87000$ due to demand parameter but due to the changes of vendor's production rate this range lies near $\$ 65000$ exclusively.

Table 7 gives a sensitivity analysis for the change of the expiry time parameter $L$ on and from $(-30 \%,-20 \%$, $-10 \%, 0 \%,+10 \%,+20 \%$ and $+30 \%)$ exclusively. We observe that the longer the product's shelf life, the longer the vendor's shipping quantity as well as buyer's order quantity and both optimal profit for buyer, vendor and the whole system profit that gets range $\$ 65400-\$ 67000$ (shown in Fig. 9) with respect to the learning frequency 4. Also, we notice that, if all the other parameters remain unchanged, a shorter product's shelf life always results in a lower the profit for the vendor and buyer as well as the whole system profit.

\section{Conclusion}

This study investigated an integrated vendor-buyer inventory model for deteriorating items (especially on COVID-19 medical materials) with expiration dates in a JIT environment that accounted for environmental pollution due to production. Profit functions for the vendor, the buyer and the integrated supply chain are derived. We have estimated first a pollution index via production modeling then the main COVID-19 material production model has been discussed. Since the demand patters vary with the learning experiences of the decision maker, so we have fuzzified the demand rate assuming triangular dense fuzzy set. To solve the model a new defuzzification method has been employed. The numerical results are compared with the crisp for centralized 
and decentralized mode of conduct and that for centralized fuzzy system. As per research questions, our findings reveal that we definitely may estimate the actual pollution function for a particular type of product efficiently. In decentralized crisp policy, the joint profit reaches to $\$ 54630.41$ but that for centralized model it becomes $\$ 65009.91$ which is almost $19 \%$ higher than that of earlier value with respect to the optimum cycle time of the buyer is 0.0980 months, vendor's cycle time is 0.3152 months for the optimum order quantity is 249.083 units with 4 successive shipments respectively.

However, the dense fuzzy centralized model having 4 learning frequencies, the profit of the chain are raised to $\$ 66344.05$ which is almost $2.01 \%$ extra gains of the decision maker. In this case, the order quantity assumes value 249.92 units with respect to the buyer's cycle time gets value near 0.1 month and that for vendor it becomes 0.32 month respectively. In the whole study we see dense fuzzy system is much suitable for the decision maker to adopt the best strategic policy because in this case $2.1 \%$ more profit can be gained on the basis of the same cycle time and other infrastructures. More over this study is limited to the fuzzification of the demand parameter only. Fuzzifying other parameters like cost components may be put as our future work.

\section{Appendix A.}

To solve the problem, we apply the following theoretical result in generalized concave functions. Lemma 1: If $f(x)$ is non-negative, differentiable and (strictly) concave, and $g(x)$ is positive, differentiable and convex, then the real-value function

$$
z(x)=\frac{f(x)}{g(x)}
$$

is (strictly) pseudo-concave. For detailed proof, please see Cambini and Martein ([5], p. 245).

Let

$$
\mathrm{TP}_{b}\left(T_{b}\right)=\frac{F\left(T_{b}\right)}{G\left(T_{b}\right)}
$$

where

$$
\begin{aligned}
F\left(T_{b}\right)= & s \mathrm{DT}_{b}-\left[A_{b}+c_{T}+\left(v+c_{t}\right) D(1+L) \log \left(\frac{1+L}{1+L-T_{b}}\right)\right] \\
& -h_{b} D\left[\frac{1}{2}\left(1+L^{2}\right) \log \left(\frac{1+L}{1+L-T_{b}}\right)+L \log \left(\frac{1+L}{1+L-T_{b}}\right)-\frac{1}{2} T_{b}-\frac{1}{2} L T_{b}+\frac{1}{4} T_{b}^{2}\right]
\end{aligned}
$$

and $G\left(T_{b}\right)=T_{b}>0$. Now, the first and second order derivatives of $F\left(T_{b}\right)$ w.r.t. $T_{b}$ can be written as

$$
\frac{\mathrm{d} F\left(T_{b}\right)}{\mathrm{d} T_{b}}=s D-\left(v+C_{t}\right) D(1+L) \frac{1}{1+L-T_{b}}-h_{b} D\left[\frac{1+L^{2}}{2\left(1+L-T_{b}\right)}+\frac{L}{\left(1+L-T_{b}\right)}-\frac{1}{2}-\frac{L}{2}+\frac{T_{b}}{2}\right]
$$

and

$$
\frac{\mathrm{d}^{2} F\left(T_{b}\right)}{\mathrm{d} T_{b}^{2}}=-\frac{\left(v+C_{t}\right) D(1+L)}{\left(1+L-T_{b}\right)^{2}}-h_{b} D\left[\frac{1}{2}+\frac{L}{\left(1+L-T_{b}\right)^{2}}+\frac{1+L^{2}}{2\left(1+L-T_{b}\right)^{2}}\right]<0 .
$$

Therefore, $\operatorname{TP}_{b}\left(T_{b}\right)=\frac{F\left(T_{b}\right)}{G\left(T_{b}\right)}$ is strictly pseudo-concave function in $T_{b}$.

\section{Appendix B.}

Proof of Proposition 4.2. The vendor's annual profit function per unit time is

$$
\begin{aligned}
\operatorname{TP}_{v}\left(m, T_{v}\right)= & \frac{1}{T_{v}+T_{b}}\left\{v m D(1+L) \log \left[\frac{1+L}{1+L-T_{b}}\right]-A_{v}-c P(1+L)\left[1-e^{\frac{-m q}{P\left(1+L-T_{v}\right)}}\right]\right. \\
& -h_{v} P\left[\frac{\log (1+L)}{2}\left\{(1+L)^{2}-\left(1+L-T_{s}\right)^{2}\right\}\right]
\end{aligned}
$$


INVENTORY DECISION FOR PRODUCTS WITH DETERIORATION AND EXPIRATION DATES

$$
\begin{aligned}
& +h_{v} P\left[\frac{(1+L)^{2}}{2}\left\{\log (1+L)-\frac{1}{2}\right\}-\frac{1}{2}\left(1+L-T_{s}\right)^{2}\left\{\log \left(1+L-T_{s}\right)-\frac{1}{2}\right\}\right] \\
& -h_{v} \frac{m q}{\left(1+L-T_{v}\right)}\left[\left\{(1+L) T_{v}-\frac{T_{v}^{2}}{2}\right\}-\left\{(1+L) T_{s}-\frac{T_{s}^{2}}{2}\right\}\right] \\
& +h_{v} T_{b} \frac{m(m-1)}{2} D(1+L) \log \left[\frac{1+L}{1+L-T_{b}}\right] \\
& \left.-p_{c}\left[\left(m T_{v}-\frac{\left(1-e^{-\varphi m T_{v}}\right)}{\varphi}\right) \frac{\gamma p\left(p^{m}-1\right)}{p-1}+\frac{\left(1-e^{-\varphi m T_{v}}\right)}{\varphi} \frac{\gamma^{\prime}\left(p_{0}^{m}-1\right)}{p_{0}-1}\right]\right\} .
\end{aligned}
$$

After substituting $T_{v}=T_{p}+(m-1) T_{b}$ in the above profit function $\operatorname{TP}_{v}\left(m, T_{v}\right)$ reduces to $\operatorname{TP}_{v}(m)$ and which can be written as

$$
T p_{v}(m)=\frac{J(m)}{H(m)}
$$

where

$$
H(m)=T_{p}+m T_{b}>0
$$

and

$$
\begin{aligned}
J(m)= & \left\{v m D(1+L) \log \left[\frac{1+L}{1+L-T_{b}}\right]-A_{v}-c P(1+L)\left[1-e^{\frac{-m q}{P\left(1+L-T_{v}\right)}}\right]\right. \\
& -h_{v} P\left[\frac{\log (1+L)}{2}\left\{(1+L)^{2}-\left(1+L-T_{s}\right)^{2}\right\}\right] \\
& +h_{v} P\left[\frac{(1+L)^{2}}{2}\left\{\log (1+L)-\frac{1}{2}\right\}-\frac{1}{2}\left(1+L-T_{s}\right)^{2}\left\{\log \left(1+L-T_{s}\right)-\frac{1}{2}\right\}\right] \\
& -h_{v} \frac{m q}{\left(1+L-T_{v}\right)}\left[\left\{(1+L) T_{v}-\frac{T_{v}^{2}}{2}\right\}-\left\{(1+L) T_{s}-\frac{T_{s}^{2}}{2}\right\}\right] \\
& +h_{v} T_{b} \frac{m(m-1)}{2} D(1+L) \log \left[\frac{1+L}{1+L-T_{b}}\right] \\
& \left.-p_{c}\left[\left(m T_{v}-\frac{\left(1-e^{-\varphi m T_{v}}\right)}{\varphi}\right) \frac{\gamma p\left(p^{m}-1\right)}{p-1}+\frac{\left(1-e^{-\varphi m T_{v}}\right)}{\varphi} \frac{\gamma^{\prime}\left(p_{0}^{m}-1\right)}{p_{0}-1}\right]\right\} .
\end{aligned}
$$

Also,

$$
T_{v}=T_{p}+(m-1) T_{b} .
$$

Using (A.1) and $\frac{\mathrm{d} T_{v}}{\mathrm{~d} m}=T_{b}$, the first order derivative with respect to $m$ can be written as

$$
\begin{aligned}
\frac{\mathrm{d} J(m)}{\mathrm{d} m}= & v D(1+L) \log \left(\frac{1+L}{1+L-T_{b}}\right)-\frac{c(1+L) m q T_{b}}{\left(1+L-T_{v}\right)^{2}} e^{-\left(\frac{m q}{P\left(1+L-T_{v}\right)}\right)}-h_{v} q\left[\frac{m T_{b}}{\left(1+L-T_{v}\right)^{2}}\right. \\
& \left.\times\left[\left\{(1-L) T_{v}-\frac{T_{v}^{2}}{2}\right\}-\left\{(1+L) T_{s}-\frac{T_{s}^{2}}{2}\right\}\right]\right]+\frac{1}{\left(1+L-T_{v}\right)}\left[\left\{(1+L) T_{v}-\frac{T_{v}^{2}}{2}\right\}\right. \\
& \left.\left.-\left\{(1+L) T_{s}-\frac{T_{s}^{2}}{2}\right\}\right]+\frac{m}{\left(1+L-T_{v}\right)}(1+L) T_{b}-T_{b} T_{v}\right]+h_{v} T_{b} D(1+L) \log \left(\frac{1+L}{1+L-T_{b}}\right) \\
& \times\left(\frac{2 m-1}{2}\right)-P_{c}\left[\frac{\gamma p\left(p^{m}-1\right)}{(p-1)}\left\{\left(T_{v}+m T_{b}\right)\left(1-e^{-\phi m T_{v}}\right)\right\}+\left(m T_{v}-\left(\frac{1-e^{-\phi m T_{v}}}{\phi}\right)\right)\right.
\end{aligned}
$$


498

M. CHOUDHURY ET AL.

$$
\left.\times \frac{\gamma p}{p-1} p^{m} \log p+\frac{\gamma^{\prime}\left(p_{0}^{m}-1\right)}{\left(p_{0}-1\right)}\left(m T_{b}+T_{v}\right) e^{-\phi m T_{v}}+\frac{\left(1-e^{-\phi m T_{v}}\right)}{\phi} \frac{\gamma^{\prime}}{\left(p_{0}-1\right)} p_{0}^{m} \log p_{0}\right]
$$

and the second order derivative of $J(m)$ with respect to $m$ can be written as

$$
\begin{aligned}
\frac{\mathrm{d}^{2} J(m)}{\mathrm{d} m^{2}}= & -c q T_{b}(1+L)\left[\frac{e^{-\left(\frac{m q}{P\left(1+L-T_{v}\right)}\right)}}{\left(1+L-T_{v}\right)^{2}}+\frac{2 m T_{b}}{\left(1+L-T_{v}\right)^{3}} e^{-\left(\frac{m q}{P\left(1+L-T_{v}\right)}\right)}-\frac{m q}{P\left(1+L-T_{v}\right)^{2}} e^{-\left(\frac{m q}{P\left(1+L-T_{v}\right)}\right)}\right. \\
& \left.\times\left\{\frac{m T_{b}}{\left(1+L-T_{v}\right)^{2}}+\frac{1}{\left(1+L-T_{v}\right)}\right\}\right]-h_{v} q\left[\frac { 2 T _ { b } } { ( 1 + L - T _ { v } ) ^ { 2 } } \left(\left\{(1+L) T_{v}-\frac{T_{v}^{2}}{2}\right\}\right.\right. \\
& \left.-\left\{(1+L) T_{s}-\frac{T_{s}^{2}}{2}\right\}\right)+\frac{2 m T_{b}^{2}}{\left(1+L-T_{v}\right)^{3}}\left(\left\{(1+L) T_{v}-\frac{T_{v}^{2}}{2}\right\}-\left\{(1+L) T_{s}-\frac{T_{s}^{2}}{2}\right\}\right) \\
& \left.+\frac{2 m T_{b}}{\left(1+L-T_{v}\right)^{2}}\left\{(1+L) T_{b}-T_{b} T_{v}\right\}+\frac{2}{\left(1+L-T_{v}\right)}\left\{(1+L) T_{b}-T_{b} T_{v}\right\}-\frac{m T_{b}^{2}}{\left(1+L-T_{v}\right)}\right] \\
& +h_{v} T_{b} D(1+L) \log \left(\frac{1+L}{1+L-T_{b}}\right)-p_{c}\left[\frac{\gamma p\left(p^{m}-1\right)}{(p-1)}\left[2 T_{b}\left(1-e^{-\phi m T_{v}}\right)+\phi T_{b}\left(T_{v}+m T_{b}\right)^{2} e^{-\phi m T_{v}}\right]\right. \\
& +\frac{2 \gamma p}{p-1} p^{m}\left(\log p_{0}\right)\left[\left(T_{v}+m T_{b}\right)\left(1-e^{-\phi m T_{v}}\right)\right]+\frac{\gamma p}{p-1} p^{m}\left(\log p_{0}\right)^{2}\left(m T_{v}-\left(\frac{1-e^{-\phi m T_{v}}}{\phi}\right)\right) \\
& +\frac{\gamma^{\prime}}{\left(p_{0}-1\right)} p_{0}^{m}\left(\log p_{0}\right)\left(T_{v}+m T_{b}\right) e^{-\phi m T_{v}}+\frac{2 \gamma^{\prime} T_{b}}{\left(p_{0}-1\right)}\left(p_{0}^{m}-1\right) e^{-\phi m T_{v}}-\frac{\gamma^{\prime} \phi}{\left(p_{0}-1\right)}\left(p_{0}^{m}-1\right) \\
& \times e^{-\phi m T_{v}}\left(m T_{b}+T_{v}\right)^{2}+\frac{\gamma^{\prime}\left(\log p_{0}\right)}{\left(p_{0}-1\right) \phi}\left[p_{0}^{m}\left(\log p_{0}\right)\left(1-e^{-\phi m T_{v}}\right)+\phi p_{0}^{m}\left(T_{v}+m T_{b}\right) e^{-\phi m T_{v}}\right] .
\end{aligned}
$$

This can be rewritten as

$$
\begin{aligned}
\frac{\mathrm{d}^{2} J(m)}{\mathrm{d} m^{2}}= & \left.-c q T_{b}(1+L) e^{-\left(\frac{m q}{P\left(1+L-T_{v}\right.}\right)}\right)\left[\frac{m}{P\left(1+L-T_{v}\right)^{3}}\left(2 P T_{b}-q\right)+\frac{1}{P\left(1+L-T_{v}\right)^{4}}\right. \\
& \left.\times\left\{P\left(1+L-T_{v}\right)^{2}-m^{2} q T_{b}\right\}\right]-h_{v} q\left[\frac{2 T_{b}}{\left(1+L-T_{v}\right)^{2}}\left(\left\{(1+L) T_{v}-\frac{T_{v}^{2}}{2}\right\}-\left\{(1+L) T_{s}-\frac{T_{s}^{2}}{2}\right\}\right)\right. \\
& \left.+\frac{2 m T_{b}^{2}}{\left(1+L-T_{v}\right)^{3}}\left(\left\{(1+L) T_{v}-\frac{T_{v}^{2}}{2}\right\}-\left\{(1+L) T_{s}-\frac{T_{s}^{2}}{2}\right\}\right)+\frac{m T_{b}^{2}}{\left(1+L-T_{v}\right)}\right] \\
& -h_{v} q T_{b}-p_{c}\left[\frac{\gamma p\left(p^{m}-1\right)}{(p-1)} 2 T_{b}\left(1-e^{-\phi m T_{v}}\right)+\phi\left(T_{v}+m T_{b}\right)^{2} e^{-\phi m T_{v}}\left(T_{b} \frac{\gamma p\left(p^{m}-1\right)}{(p-1)}\right.\right. \\
& \left.-\gamma^{\prime} \frac{\left(p_{0}^{m}-1\right)}{\left(p_{0}-1\right)}\right)+2 \frac{2 \gamma p}{p-1} p^{m}(\log p)\left[\left(T_{v}+m T_{b}\right)\left(1-e^{-\phi m T_{v}}\right)\right]+\frac{\gamma p}{p-1}(\log p)^{2}\left(m T_{v}-\frac{1-e^{-\phi m T_{v}}}{\phi}\right) \\
& +\frac{\gamma^{\prime}}{\left(p_{0}-1\right)} p_{0}^{m}\left(\log p_{0}\right)\left(T_{v}+m T_{b}\right) e^{\phi m T_{v}}+\frac{2 \gamma^{\prime} T_{b}}{\left(p_{0}-1\right)}\left(p_{0}^{m}-1\right) e^{\phi m T_{v}}+\frac{\gamma^{\prime}\left(\log p_{0}\right)}{\left(p_{0}-1\right) \phi} \\
& \times\left[p_{0}^{m}\left(\log p_{0}\right)\left(1-e^{\phi m T_{v}}\right)+\phi p_{0}^{m}\left(T_{v}+m T_{b}\right) e^{\phi m T_{v}}\right] .
\end{aligned}
$$

Since all terms confined in the third brackets of the above expression (B.4) are positive, so always we have $\frac{d^{2} J(m)}{d m^{2}}<0$. Therefore, applying Lemma 1 (Cambini and Martein [5]'s theorem), the vendor's profit function per unit time, $\operatorname{TP}_{v}(m)$, is strictly pseudo-concave with respect to $m$ and hence $\operatorname{TP}_{v}(m)$ has a global maximum at the point, $m=m^{*}(\in(0, \infty))$. 


\section{Appendix C.}

Proof of Proposition 4.3. Since the profit function of the supply chain is the sum of the retailer's and the vendor's profit functions, so, according to Proposition 4.1, it is clear that the retailer's profit function is pseudo-concave with respect to $T_{b}$. However, from the Proposition 4.2 it is clear that the vendor's profit function pseudo-concave with respect to $m$. Thus, we can conclude that the profit function of the supply chain having the sum of two pseudo-concave functions is also pseudo-concave with respect to $T_{b}$ and $m$ exclusively.

Acknowledgements. The authors are grateful to the Editor-in-chief, Associate editors and anonymous reviewers for their valuable comments and suggestions to improve the quality of this article.

\section{REFERENCES}

[1] T. Allahviranloo and R. Saneifard, Defuzzification method for ranking fuzzy numbers based on center of gravity. Iran. J. Fuzzy Syst. 9 (2012) 57-67.

[2] A. Banerjee, A joint economic-lot-size model for purchaser and vendor. Decis. Sci. 17 (1986) 292-311.

[3] R.E. Bellman and L.A. Zadeh, Decision making in a fuzzy environment. Manag. Sci. 17 (1970) B141-B164.

[4] S. Benjaafar, Y. Li and M. Daskin, Carbon footprint and the management of supply chain: insights from simple models. IEEE Trans. Autom. Sci. Eng. 10 (2013) 99-116.

[5] A. Cambini and L. Martein, Generalized convexity and optimization: Theory and applications. Berlin, Heidelberg: SpringerVerlag (2009)

[6] S.K. De and I. Beg, Triangular dense fuzzy sets and new defuzzification methods. J. Intell. Fuzzy Syst. 31 (2016) $469-477$.

[7] S.K. De and G.C. Mahata, Decision of a fuzzy inventory with fuzzy backorder Model under cloudy fuzzy demand rate. Int. J. Appl. Comp. Math. 3 (2017) 2593-2609.

[8] S.K. De and S.S. Sana, Backlogging EOQ model for promotional effort and selling price sensitive demand- an intuitionistic fuzzy approach. Ann. Oper. Res. 233 (2015) 57-76.

[9] R. Ezzati, T. Allahviranloo, S. Khezerloo and M. Khezerloo, An approach for ranking of fuzzy numbers. Exp. Syst. App. 39 (2012) 690-695.

[10] P.M. Ghare and S.F. Schrader, A model for exponentially decaying inventory. J. Ind. Eng. 14 (1963) 238.

[11] B.C. Giri and B. Roy, A vendor-buyer integrated production-inventory model with quantity discount and unequal sized shipments. Int. J. Prod. Res. 16 (2013) 1-13.

[12] S.K. Goyal, An integrated inventory model for a single supplier-single customer problem. Int. J. Prod. Res. 15 (1976) $107-111$.

[13] S.K. Goyal, A joint economic-lot-size model for purchaser and vendor: a comment. Decis. Sci. 19 (1988) $236-241$.

[14] S.K. Goyal, A one-vendor multi-buyer integrated inventory model: a comment. Eur. J. Oper. Res. 82 (1995) 209-210.

[15] S.K. Goyal and B.C. Giri, Recent trends in modeling of deteriorating inventory. Eur. J. Oper. Res. 134 (2001) 1-16.

[16] S.K. Goyal and F. Nebebe, Determination of economic production-shipment policy for a single-vendor-single-buyer system. Eur. J. Oper. Res. 121 (2000) 175-178.

[17] T. Hajjari and S. Abbasbandy, A note on "The revised method of ranking LR fuzzy number based on deviation degree". Exp. Syst. Appl. 39 (2011) 13491-13492.

[18] P. He, W. Zhang, X. Xu and Y. Bian, Production lot-sizing and carbon emissions under cap-and trade and carbon tax regulations. J. Clean. Prod. 103 (2015) 241-248.

[19] L. He, J. Mao, C. Hu and Z. Xiao, Carbon emission regulation and operations in the supply chain super network under stringent carbon policy. J. Clean. Prod. 238 (2019) 117652.

[20] R.M. Hill, The single-vendor single-buyer integrated production-inventory model with a generalized policy. Eur. J. Oper. Res. 97 (1997) 493-499.

[21] S.M. Hosseini-Motlagh, M. Nouri-Harzvili and R. Zirakpourdehkordi, Two-level supply chain quality improvement through a whole sale price coordination contract on pricing, quality and services. Int. J. Ind. Eng. Prod. Res. 30 (2019) $287-312$.

[22] V. Hovelaque and L. Bironneau, The carbon-constrained EOQ model with carbon emission dependent demand. Int. J. Prod. Econ. 164 (2015) 285-291.

[23] G. Hua, T.C.E. Cheng and S. Wang, Managing carbon footprints in inventory management. Int. J. Prod. Econ. 132 (2011) $178-185$

[24] N. Kazemi, E.U. Olugu, A.R. SalwaHanim and R. Ghazilla, A fuzzy EOQ model with backorders and forgetting effect on fuzzy parameters: an empirical study. Comp. Ind. Eng. 96 (2016) 140-148.

[25] P. Kelle, F. Al-khateeb and P.A. Miller, Partnership and negotiation support by joint optimal ordering/setup policies for JIT. Int. J. Prod. Econ. 81-82 (2003) 431-441.

[26] A. Kumar, P. Singh, P. Kaur and A. Kaur, A new approach for ranking of L-R type generalized fuzzy numbers. Exp. Syst. Appl. 38 (2011) 10906-10910.

[27] Y.J. Lin and C.H. Ho, Integrated inventory model with quantity discount and price-sensitive demand. TOP 19(2011) 177-188.

[28] L. Lu, A one-vendor multi-buyer integrated inventory model. Eur. J. Oper. Res. 81 (1995) 312-323. 
[29] G.C. Mahata, A production-inventory model with imperfect production process and partial backlogging under learning considerations in fuzzy random environments. J Intell. Manuf. 28 (2017) 883-897.

[30] G.C. Mahata and A. Goswami, An EOQ model for deteriorating items under trade credit financing in the fuzzy sense. Prod. Plan. Cont. 18 (2007) 681-692.

[31] G.C. Mahata and P. Mahata, Analysis of a fuzzy economic order quantity model for deteriorating items under retailer partial trade credit financing in a supply chain. Math. Comp. Model. 53 (2011) 1621-1636.

[32] C. Mahato and G.C. Mahata, Optimal inventory policies for deteriorating items with expiration date and dynamic demand under two-level trade credit. Opsearch 58 (2021) 994-1017.

[33] C. Mahato and G.C. Mahata, Sustainable ordering policies with capacity constraint under order-size-dependent trade credit, all-units discount, carbon emission, and partial backordering. Process. Integr. Optim. Sustain. 5 (2021) 875-903.

[34] S. Maity, A. Chakraborty, S.K. De, S.P. Mondol and S. Alam, A comprehensive study of a backlogging EOQ model with nonlinear heptagonaldensefuzzyenvironment. RAIRO-Oper. Res. 54 (2020) 267-286.

[35] A. Mukherjee and G.C. Mahata, Optimal replenishment and credit policy in an inventory model for deteriorating items under two-levels of trade credit policy when demand depends on both time and credit period involving default risk. RAIRO-Oper. Res. 52 (2018) 1175-1200.

[36] P. Muniappan, R. Uthayakumar and S. Ganesh, A production inventory model for vendor-buyer coordination with quantity discount, backordering and rework for fixed life time products. J. Int.Prod. Econ. 33 (2016) 355-362.

[37] M. Nouri, S.M. Hosseini-Motlagh and M. Nematollahi, Proposing a discount policy for two-level supply chain coordination with periodic review replenishment and promotional efforts decisions. Oper. Res. 21 (2021) 365-398.

[38] L.Y. Ouyang, C.H. Ho, C.H. Su and C.T. Yang, An integrated inventory model with capacity constraint and order-size dependent trade credit. Comput. Ind. Eng. 84 (2015) 133-143.

[39] S. Panja and S.K. Mondal, Analyzing a four-layer green supply chain imperfect production inventory model for green products under type-2 fuzzy credit period. Comput. Ind. Eng. 129 (2019) 435-453.

[40] F. Raafat, Survey of literature on continuously deteriorating inventory model. J. Oper. Res. Soc. 42 (1991) $27-37$.

[41] H. Rau, M.Y. Wu and H.M. Wee, Integrated inventory model for deteriorating items under a multi-echelon supply chain environment. Int. J. Prod. Econ. 86 (2003) 155-162.

[42] Y.J. Shen, K.F. Shen and C.T. Yang, A production-inventory model for deteriorating items with collaborative preservation technology investment under carbon tax. Sustainability 11 (2019) 5027.

[43] E. Teimoury and S.M.M. Kazemi, An integrated pricing and inventory model for deteriorating products in a two stage supply chain under replacement and shortage. Sci. Iran. 24 (2017) 342-354.

[44] W.C. Wang, J.T. Teng and K.R. Lou, Seller's optimal credit period and cycle time in a supply chain for deteriorating items with maximum lifetime. Eur. J. Oper. Res. 232 (2014) 315-321.

[45] I.P. Wright, Factors affecting the cost of airplanes. J. Aeronaut. Sci. 3 (1936) 122-128.

[46] J. Wu, C.T. Chang, J.T. Teng and K.K. Lai, Optimal order quantity and selling price over a product life cycle with deterioration rate linked to expiration date. Int. J. Prod. Econ. 193 (2017) 343-351.

[47] R.R. Yager, A procedure for ordering fuzzy subsets of the unit interval. Inf. Sci. 24 (1981) 143-161.

[48] V.F. Yu, H.T.X. Chi, L.Q. Dat, P.N.K. Phuc and C.W. Shen, Ranking generalized fuzzy numbers in fuzzy decision making based on the left and right transfer coefficients and areas. Appl. Math. Model. 37 (2013) 8106-8117.

[49] L.A. Zadeh, Fuzzy sets. Inf. Cont. 8 (1965) 338-356.

\section{Subscribe to Open (S2O) A fair and sustainable open access model}

This journal is currently published in open access under a Subscribe-to-Open model (S2O). S2O is a transformative model that aims to move subscription journals to open access. Open access is the free, immediate, online availability of research articles combined with the rights to use these articles fully in the digital environment. We are thankful to our subscribers and sponsors for making it possible to publish this journal in open access, free of charge for authors.

\section{Please help to maintain this journal in open access!}

Check that your library subscribes to the journal, or make a personal donation to the S2O programme, by contacting subscribers@edpsciences.org

More information, including a list of sponsors and a financial transparency report, available at: https://www. edpsciences.org/en/maths-s2o-programme 